\title{
HEREDITARY, ADDITIVE AND DIVISIBLE CLASSES IN EPIREFLECTIVE SUBCATEGORIES OF Top
}

\author{
MARTIN SLEZIAK
}

\begin{abstract}
Hereditary coreflective subcategories of an epireflective subcategory $\mathbf{A}$ of Top such that $I_{2} \notin \mathbf{A}$ (here $I_{2}$ is the 2-point indiscrete space) were studied in 4. It was shown that a coreflective subcategory $\mathbf{B}$ of $\mathbf{A}$ is hereditary (closed under the formation of subspaces) if and only if it is closed under the formation of prime factors. The main problem studied in this paper is the question whether this claim remains true if we study the (more general) subcategories of $\mathbf{A}$ which are closed under topological sums and quotients in $\mathbf{A}$ instead of the coreflective subcategories of $\mathbf{A}$.

We show that this is true if $\mathbf{A} \subseteq$ Haus or under some reasonable conditions on B. E.g., this holds if $\mathbf{B}$ contains either a prime space, or a space which is not locally connected, or a totally disconnected space or a non-discrete Hausdorff space.

We touch also other questions related to such subclasses of $\mathbf{A}$. We introduce a method extending the results from the case of non-bireflective subcategories (which was studied in 44) to arbitrary epireflective subcategories of Top. We also prove some new facts about the lattice of coreflective subcategories of Top and $\mathbf{Z D}$.

Keywords: epireflective subcategory, coreflective subcategory, hereditary subcategory, prime factor, prime space, bireflective subcategory, zero-dimensional spaces, $T_{0}$-spaces.

MSC2000 Primary: 54B30 and Secondary: 18B30.
\end{abstract}

\section{INTRODUCTION}

Motivated by [11, Problem 7] J. Činčura studied in [3] hereditary coreflective subcategories of the category Top of all topological spaces and continuous maps. He proved a nice characterization of hereditary coreflective subcategories using prime factors of topological spaces. However, it would be interesting to study the hereditary coreflective subcategories also in other categories of topological spaces, as Haus or, more generally, any epireflective subcategory of Top. In this case the situation becomes more complicated than in Top. For instance, in Top we obtain the hereditary coreflective hull of a coreflective subcategory $\mathbf{C}$ simply by taking the subcategory SC consisting of all subspaces of spaces from $\mathbf{C}$. This is not true in the case of coreflective subcategories of Haus anymore, as the example of $T_{2}$-subsequential spaces (see [7 or 4]) shows. The hereditary coreflective hull of Hausdorff sequential spaces in Haus are precisely the Hausdorff subsequential spaces. But not every Hausdorff subsequential space is a subspace of a Hausdorff sequential space. So the description of the hereditary coreflective hull mentioned above does not work in Haus.

Although we see that this new situation leads to some complications, in [4] it is proved that the same characterization holds if we study the same problem in an epireflective subcategory $\mathbf{A}$ of Top, which is not bireflective. Namely, it is shown 
that a coreflective subcategory of $\mathbf{A}$ is hereditary if and only if it is closed under the formation of prime factors. This paper is an attempt to study a similar situation and to add a few new results in this area of research.

We study here mainly the subcategories which are additive and divisible (i.e., closed under sums and quotient spaces) in $\mathbf{A}$. We call them briefly AD-classes. The AD-classes include coreflective subcategories as a special case. If $\mathbf{A}$ is a quotientreflective subcategory of Top (in particular if $\mathbf{A}=\mathbf{T o p}$ ), then there is no difference between these two notions. We show that in many cases an AD-class $\mathbf{B}$ in $\mathbf{A}$ is hereditary if and only if it is closed under the formation of prime factors. E.g., this holds if $\mathbf{A} \subseteq$ Haus or $\mathbf{B}$ contains at least one prime space.

We also present a method how to extend our results to bireflective subcategories of Top. (Maybe it is more precise to say that the restriction to non-bireflective subcategories is in fact not so restrictive.) For this purpose we use the correspondence between bireflective subcategories of Top and epireflective subcategories of Top consisting only of $T_{0}$-spaces. This correspondence was introduced in [19] (see also [21]).

\section{Preliminaries}

Topological terminology follows [6] with a few exceptions. We do not assume the $T_{1}$ separation axiom for zero-dimensional spaces. A neighborhood of $x$ is any set $V$ such that there exists an open subset $U$ with $x \in U \subseteq V$. (So the neighborhoods in the sense of [6] are open neighborhoods in our terminology.) Compact spaces are not necessarily Hausdorff. For the notions and results from category theory we refer to [1, in particular for reflective and coreflective subcategories of the category Top of topological spaces and continuous maps to 9 .

All subcategories are assumed to be full and isomorphism-closed. To avoid some trivial cases we assume that every subcategory of Top contains at least one space with at least two points.

By $X \prec Y$ we mean that the spaces $X$ and $Y$ have the same underlying set and $X$ has a finer topology than $Y$. By initial map we mean an initial morphism in the category Top. I.e., $f: X \rightarrow Y$ is said to be initial if $X$ has the initial topology w.r.t. $f$.

Any ordinal is the set of its predecessors ordered by $\in$. Cardinal numbers are the initial ordinals. The class of all cardinals will be denoted by $\mathrm{Cn}$.

2.1. Epireflective and coreflective subcategories. Perhaps the most important notions from category theory, which we will use in this paper, are those of reflective and coreflective subcategory. We review here some basic facts, more can be found in [1], 9] or [12.

A subcategory $\mathbf{A}$ of a category $\mathbf{B}$ is reflective if for any $\mathbf{B}$-object there exists an $\mathbf{A}$-reflection. The $\mathbf{A}$-reflection of $X \in \mathbf{B}$ is an object $R X \in \mathbf{A}$ together with a morphism $r: X \rightarrow R X$ (called the A-reflection arrow) which has the following universal property: For any morphism $f: X \rightarrow A$ with $A \in \mathbf{A}$ there exists a unique morphism $\bar{f}: R X \rightarrow A$ such that the following diagram commutes.

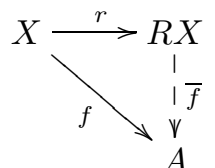


The A-reflection is determined uniquely up to homeomorphism.

The functor $R: \mathbf{B} \rightarrow \mathbf{A}$ which assigns to each $\mathbf{B}$-object its $\mathbf{A}$-reflection (and acts on morphisms in the natural way) is called a reflector. This functor is coadjoint to the embedding functor $\mathbf{A} \hookrightarrow \mathbf{B}$.

We say that $\mathbf{A}$ is epireflective (bireflective) in $\mathbf{B}$ if all $\mathbf{A}$-reflection arrows are epimorphisms (bimorphisms) in $\mathbf{B}$. If $\mathbf{B}=$ Top and all $\mathbf{A}$-reflections are quotient maps, we speak about a quotient-reflective subcategory.

A subcategory A of Top is epireflective in Top if and only if it is closed under the formation of topological products and subspaces.

By $\mathrm{EH}(\mathbf{A})$ we denote the epireflective hull of a subcategory A. A topological space $X$ belongs to $\mathrm{EH}(\mathbf{A})$ if and only if it is a subspace of a product of spaces from A. An equivalent condition is that there exist an initial monosource with domain $X$ and codomain in A. (See e.g. [19, Theorem 2] or [1, Theorem 16.8].) A similar characterization holds for bireflective hulls. A topological space $X$ belongs to the bireflective hull $\mathrm{BH}(\mathbf{A})$ of $\mathbf{A}$ if and only if there is an initial source from $X$ to $\mathbf{A}$ (15, Corollary 2] or [1, Theorem 16.8]).

By $I_{2}$ we will denote the two-point indiscrete space. An epireflective subcategory A of Top is bireflective if and only if $I_{2} \in \mathbf{A}$. Therefore $\mathrm{BH}(\mathbf{A})=\operatorname{EH}\left(\mathbf{A} \cup\left\{I_{2}\right\}\right)$.

Mostly we will work in an epireflective subcategory $\mathbf{A}$ of Top which does not contain $I_{2}$. (We will show in Section 5 how to get rid of this assumption.) The same assumption on $\mathbf{A}$ was used in [4. It is motivated by the fact that only these epireflective subcategories of Top are closed under the formation of prime factors. Under this assumption $\mathbf{A}$ is closed under topological sums, too. (Recall that we made an agreement that each subcategory contains a space with at least 2 points. Hence, A contains all discrete spaces whenever $I_{2} \notin \mathbf{A}$.)

The largest such subcategory of Top is the category $\mathbf{T o p}_{0}$ of $T_{0}$-spaces. The largest subcategory with these properties such that moreover $\mathbf{A} \subsetneq \mathbf{T o p}_{0}$ is the category $\mathbf{T o p}_{1}$ of $T_{1}$-spaces.

The smallest such subcategory of Top is the subcategory $\mathbf{Z D}_{\mathbf{0}}$ of zero-dimensional $T_{0}$-spaces. (Note that for a zero-dimensional spaces the conditions $T_{0}$ and $T_{2}$ are equivalent.) The subcategory $\mathbf{Z D}_{\mathbf{0}}$ is the epireflective hull of the 2-point discrete space $D_{2}$.

An epireflective subcategory of Top is quotient-reflective if and only if it is closed under the formation of spaces with finer topologies. In a quotient-reflective subcategory of Top regular (extremal) epimorphisms are exactly the quotient maps.

Let $\mathbf{A}$ be an epireflective subcategory of Top and $\mathbf{A} \neq \mathbf{I n d}$ (the subcategory of all indiscrete spaces). A subcategory $\mathbf{B} \subseteq \mathbf{A}$ is coreflective in $\mathbf{A}$ if and only if it is closed under topological sums and $\mathbf{A}$-extremal quotients. In particular $\mathbf{B}$ is coreflective in Top if it is closed under sums and quotients. For each subcategory $\mathbf{B}$ of $\mathbf{A}$ there exists the smallest coreflective subcategory of $\mathbf{A}$ containing $\mathbf{B}$. It is called the coreflective hull of $\mathbf{B}$ in $\mathbf{A}$ and denoted by $\mathrm{CH}_{\mathbf{A}}(\mathbf{B})$. If $\mathbf{B}=\{B\}$ consists of a single space we use the notation $\mathrm{CH}_{\mathbf{A}}(B)$. If $\mathbf{A}$ is an epireflective subcategory of Top and $\mathbf{A} \neq \mathbf{I n d}$, then the members of $\mathrm{CH}_{\mathbf{A}}(\mathbf{B})$ are exactly the $\mathbf{A}$-extremal quotients of topological sums of spaces from $\mathbf{B}$. If $\mathbf{A}=\mathbf{T o p}$, then the notation $\mathrm{CH}(\mathbf{B})$ (resp. $\mathrm{CH}(B)$ ) is used and $\mathrm{CH}(\mathbf{B})$ is called the coreflective hull of B. $\mathbf{C H}(\mathbf{B})$ is formed by quotients of topological sums of spaces from $\mathbf{B}$.

The class FG of all finitely generated spaces is the coreflective hull of all finite spaces in Top. A space is finitely generated if and only if any intersection of its open 
sets is again open. The subcategory FG is the smallest coreflective subcategory of Top containing a space, which is not a sum of indiscrete spaces, and it is the coreflective hull of the Sierpiński space $S$. The Sierpiński space $S$ is the two-point space in which only one point is isolated.

A subcategory $\mathbf{B}$ of Top is said to be hereditary, if it is closed under subspaces, and additive, if it is closed under topological sums. We say that $\mathbf{B}$ is divisible in $\mathbf{A}$ if for every quotient map $q: X \rightarrow Y$ with $X \in \mathbf{B}$ and $Y \in \mathbf{A}$ we have $Y \in \mathbf{B}$.

A class $\mathbf{B}$ which is additive and divisible in $\mathbf{A}$ will be called briefly an $A D$-class in $\mathbf{A}$. If $\mathbf{B}$ is moreover hereditary, we say that it is an $H A D$-class in $\mathbf{A}$.

We define the AD-hull (HAD-hull) of $\mathbf{B} \subseteq \mathbf{A}$ as the smallest (hereditary) ADclass in $\mathbf{A}$ containing $\mathbf{B}$. It will be denoted by $\operatorname{AD}_{\mathbf{A}}(\mathbf{B})$, resp. $\operatorname{HAD}_{\mathbf{A}}(\mathbf{B})$. It is clear that $\mathrm{AD}_{\mathbf{A}}(\mathbf{B})$ consists precisely of all spaces from $\mathbf{A}$, which are quotient spaces of topological sums of spaces from $\mathbf{B}$.

If $\mathbf{A}=$ Top or $\mathbf{A}$ is quotient-reflective in Top, then the notion of AD-class (HAD-class) coincides with the notion of (hereditary) coreflective subcategory.

Whenever $\mathbf{C}$ is coreflective in Top, the subcategory SC consisting of all subspaces of spaces from $\mathbf{C}$ is known to be coreflective as well (see e.g. [16, Remark 2.4.4(5)] or [3, Proposition 3.1]). Clearly the category $\mathrm{S}(\mathrm{CH}(\mathbf{B}))$ is the hereditary coreflective hull of $\mathbf{B}$. It will be denoted also by $\mathrm{HCH}(\mathbf{B})$. The hereditary coreflective hull of a single space $A$ in Top is denoted by $\mathrm{HCH}(A)$.

For the future reference we state some obvious relations between AD-hulls in $\mathbf{A}$ and coreflective hulls in Top in the following lemma.

Lemma 2.1. Let $\mathbf{A}$ be an epireflective subcategory of Top with $I_{2} \notin \mathbf{A}$. Then

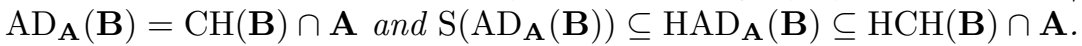

2.2. Prime spaces and prime factors. We say that a space $P$ is a prime space, if it has precisely one accumulation point $a$. All prime spaces are $T_{0}$. It is easy to see that all prime $T_{2}$-spaces are zero-dimensional.

If the point $a$ is not isolated in a subspace $P^{\prime}$ of a prime space $P$, i.e., if $P^{\prime}$ is itself a prime space, we say briefly that $P^{\prime}$ is a prime subspace of $P$.

Lemma 2.2. Let $P$ be a prime space with the accumulation point a and $P^{\prime}$ be a prime subspace of $P$. Then the map $f: P \rightarrow P^{\prime}$, such that $f(x)=x$ if $x \in P^{\prime}$ and $f(x)=a$ otherwise is a retraction.

The fact that the discrete spaces form the smallest coreflective subcategory Disc of Top together with Lemma 2.2 imply that for any prime space $P$ all its subspaces are contained in $\mathrm{CH}(P)$. (They are moreover contained in $\mathrm{CH}_{\mathbf{A}}(P)$ for any epireflective subcategory $\mathbf{A}$ of Top with $I_{2} \notin \mathbf{A}$.)

For any space $X$ and any point $a \in X$ we define the prime factor of $X$ at $a$ as the topological space on the same set in which all points different from $a$ are isolated and the neighborhoods of $a$ are the same as in the original topology. Clearly, $X_{a}$ is a discrete or prime space.

Note that a prime space is $T_{2}$ if and only if it is $T_{1}$. Thus a prime factor of a $T_{1}$-space is either a prime $T_{2}$-space or a discrete space.

Each topological space $X$ is a quotient of the sum of all its prime factors. The quotient map is obtained simply by mapping a point $x$ in a summand $X_{a}$ to the same point $x$ of the space $X$. 


\section{HEREDITY AND PRIME FACTORS}

Hereditary coreflective subcategories of Top were studied in 3. In this paper we are interested in a slightly more general situation - we use an epireflective subcategory $\mathbf{A}$ (with $I_{2} \notin \mathbf{A}$ ) instead of Top. There are two natural generalizations of coreflective subcategories - we can study coreflective subcategories of $\mathbf{A}$ or ADclasses in $\mathbf{A}$. (Both of them were already studied in 44, AD-classes only in two special cases $\mathbf{A}=\mathbf{Z D}_{\mathbf{0}}$ and $\mathbf{A}=\mathbf{T y c h}$.)

Clearly, every coreflective subcategory $\mathbf{B}$ of $\mathbf{A}$ is an AD-class in $\mathbf{A}$. (The opposite implication does not hold. The counterexample is the subcategory of $k$-spaces in Tych. It is the AD-hull of compact spaces in Tych, but the coreflective hull of compact spaces in Tych is the larger subcategory of $k_{R^{-}}$-spaces. For more details see Example 3.18 at the end of this section.)

It was shown in 3 , that a coreflective subcategory of Top different from $\mathrm{CH}(\mathbf{I n d})$ (the coreflective hull of indiscrete spaces) is hereditary if and only if it is closed under the formation of prime factors. The same result was shown in 4 for coreflective subcategories of $\mathbf{A}$ (A being an epireflective subcategory of Top with $I_{2} \notin \mathbf{A}$ ) and for AD-classes in $\mathbf{Z D}_{\mathbf{0}}$ and $\mathbf{T y c h}$. We would like to generalize this result for AD-classes in more epireflective subcategories.

The main results of this section are Theorem 3.10 and its consequences. They say that an AD-class is hereditary if and only if it is closed under prime factors whenever this AD-class contains a prime space (or a space with "good" properties). Using this fact we can show in Theorem 3.14 another interesting result: If $\mathbf{A} \subseteq$ Haus, then an AD-class in $\mathbf{A}$ is hereditary if and only if it is closed under prime factors. So if we work only with Hausdorff spaces, the desired equivalence between heredity and closedness under prime factors is true. In section 6 we try to find some other cases when this statement holds.

3.1. When heredity implies closedness under prime factors? It is easy to show that for an AD-class closedness under prime factors implies heredity. The proof follows the proof of [4, Theorem 2, Theorem 7].

Lemma 3.1. Let $\mathbf{B}$ be additive and divisible in $\mathbf{A}, \mathbf{A}$ being an epireflective subcategory of Top with $I_{2} \notin \mathbf{A}$. If $\mathbf{B}$ is closed under prime factors, then it is hereditary.

Proof. Let $X \in \mathbf{B}$ and $Y$ be a nonempty subspace of $X$. We want to show that $Y \in \mathbf{B}$. Let $a \in Y$. The prime factor $Y_{a}$ is a subspace of the corresponding prime factor $X_{a}$ of $X$. Since $\mathbf{B}$ is closed under prime factors, $X_{a} \in \mathbf{B}$ and, by Lemma 2.2. $Y_{a} \in \mathbf{B}$ as well. Since $Y \in \mathbf{A}$ and it is a quotient space of all its prime factors, $Y \in \mathbf{B}$.

This paper is mostly devoted to the effort to show that the opposite implication holds too (under some assumptions on $\mathbf{A}$ or $\mathbf{B}$ ).

We first need to define the space $X \triangle_{b} Y$ which was used in a similar context in [4.

Definition 3.2. If $X$ and $Y$ are topological spaces, $b \in Y$ and $\{b\}$ is closed in $Y$, then we denote by $X \triangle_{b} Y$ the topological space on the set $X \times Y$ which has the final topology w.r.t the family of maps $\left\{f, g_{a} ; a \in X\right\}$, where $f: X \rightarrow X \times Y$, $f(x)=(x, b)$ and $g_{a}: Y \rightarrow X \times Y, g_{a}(y)=(a, y)$. 
In other words, $X \triangle_{b} Y$ is the quotient of $X \sqcup\left(\coprod_{a \in X} Y\right)$ with respect to the map obtained as the combination of the maps $f$ and $g_{a}, a \in X$. Since the space $X \triangle_{b} Y$ is constructed from $X$ and $Y$ using only topological sums and quotient maps, any coreflective subcategory of Top containing $X$ and $Y$ contains $X \triangle_{b} Y$, too.

A local base for the topology of $X \triangle_{b} Y$ at a point $(a, y), y \neq b$, is $\{\{a\} \times V ; V$ is an open neighborhood of $y$ in $Y\}$. A local base at $(a, b)$ consists of all sets of the form $\bigcup_{x \in U}\{x\} \times V_{x}$ where $U$ is an open neighborhood of $a$ in $X$ and each $V_{x}$ is an open neighborhood of $b$ in $Y$.

Figure 1 depicts the space $X \triangle_{b} Y$ by showing typical sets from the neighborhood basis.

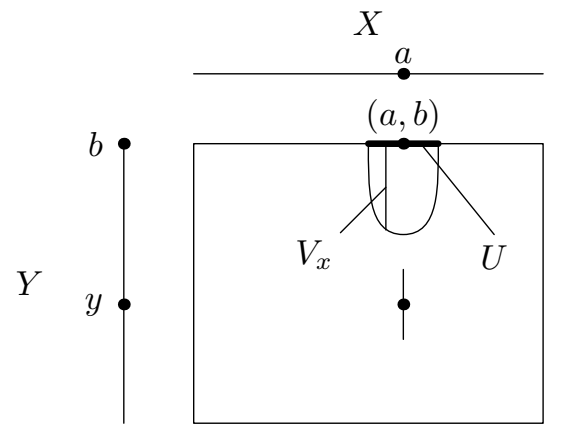

Figure 1. The space $X \triangle_{b} Y$

Let $X_{(Y, b)}^{a}$ be the subspace of $X \triangle_{b} Y$ on the subset $\{(a, b)\} \cup(X \backslash\{a\}) \times(Y \backslash\{b\})$ and $X_{a}$ be the prime factor of $X$ at $a$. It was shown in [4] that, for any space $Y$ in which the subset $\{b\}$ is closed but not open, the map $q: X_{(Y, b)}^{a} \rightarrow X_{a}$ given by $q(x, y)=x$ is quotient. This yields the following proposition:

Proposition 3.3. Let $\mathbf{B}$ be an HAD-class in an epireflective subcategory $\mathbf{A}$ of $\mathbf{T o p}$ with $I_{2} \notin \mathbf{A}$. Let for any $X \in \mathbf{B}$ there exist $Y \in \mathbf{B}$ and a non-isolated point $b \in Y$ with $\{b\}$ being closed in $Y$ such that $X \triangle_{b} Y$ belongs to $\mathbf{A}$. Then $\mathbf{B}$ is closed under the formation of prime factors.

Proof. Let $X \in \mathbf{B}$ and $a \in X$. We want to show that $X_{a} \in \mathbf{B}$. By the assumption there exists $Y \in \mathbf{A}$ and $b \in Y$ such that $\{b\}$ is closed but not open and $X \triangle_{b} Y \in \mathbf{A}$.

Since $X \triangle_{b} Y$ is constructed using quotients and sums, we get $X \triangle_{b} Y \in \mathbf{B}$, as well. Therefore also its subspace $X_{(Y, b)}^{a}$ belongs to $\mathbf{B}$ and $X_{a}$ is a quotient of this space.

Definition 3.4. We say that a subcategory $\mathbf{A}$ of Top is closed under $\triangle$ if $X \triangle_{b} Y \in$ A whenever $X, Y \in \mathbf{A}$ and $b \in Y$.

Proposition 3.5. Let $\mathbf{A}$ be an epireflective subcategory of Top with $I_{2} \notin \mathbf{A}$. If $\mathbf{A}$ is closed under $\triangle$ and $\mathbf{B}$ is hereditary, additive and divisible in $\mathbf{A}$, then $\mathbf{B}$ is closed under prime factors.

Proof. It suffices to choose any space $Y \in \mathbf{B}$ and $b \in Y$ such that $\{b\}$ is closed and not open. (We can w.l.o.g. assume that $\mathbf{B}$ contains a non-discrete space, since discrete spaces are closed under prime factors trivially. If $\mathbf{A}=\mathbf{T o p}_{0}$, then the Sierpiński space $S$ belongs to $\mathbf{B}$ and we can take for $Y$ the space $S$. If $\mathbf{A} \neq \mathbf{T o p}_{0}$, 
then $\mathbf{A} \subseteq \mathbf{T o p}_{1}$ and in this case it suffices to take any non-discrete space for $Y$.) By Proposition 3.3 then $X_{a} \in \mathbf{B}$ whenever $a \in X \in \mathbf{B}$.

By [4, Proposition 1] every quotient-reflective subcategory of Top (in particular $\mathbf{T o p}_{0}$, Top ${ }_{1}$, Haus) is closed under the operation $\triangle$. It is also relatively easy to show that the subcategories Reg, Tych, $\mathbf{Z D}_{\mathbf{0}}$ are closed under $\triangle$.

In Example 3.17 we will show that the epireflective subcategories of Top need not be closed under $\triangle$ in general. Therefore it could be interesting to show the above result under some less restrictive conditions on $\mathbf{A}$.

Proposition 3.3 suggests that it would be useful to have some conditions on spaces $X, Y$ which imply $X \triangle_{b} Y \in \mathbf{A}$. Such a condition will be obtained in Theorem 3.10. We first introduce the operation $X \nabla_{b} Y$, which was defined in [4].

Definition 3.6. Let $X, Y$ be topological spaces and $b \in Y$ with the set $\{b\}$ closed in $Y$. The space $X \nabla_{b} Y$ is the topological space on the set $X \times Y$ which has the initial topology w.r.t. the family $h_{a}: X \times Y \rightarrow X \times Y, h_{a}(x, y)=(x, b)$ for $x \neq a$ and $h_{a}(a, y)=(a, y)$.

A subbase for this topology is formed by the sets $h_{a}^{-1}(U \times V)=(\{a\} \times V) \cup$ $(U \backslash\{a\}) \times Y$, where $a \in X, V$ is an open neighborhood of $b$ in $Y$ and $U$ is a neighborhood of $a$ in $X$, and by the sets of the form $\{a\} \times V$, where $V$ is an open set in $Y$ not containing $b$.

The space is illustrated by Figure 2

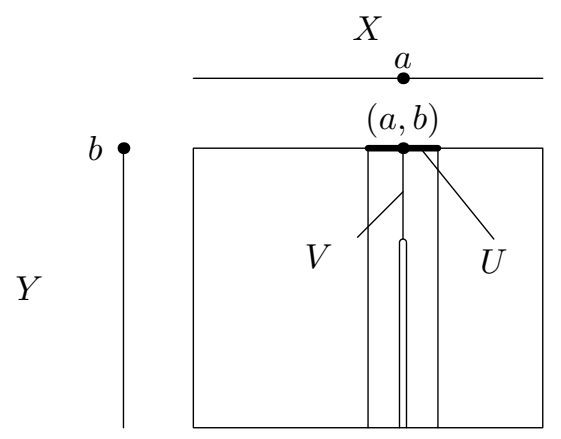

Figure 2. The space $X \nabla_{b} Y$

Observe that $X \triangle_{b} Y \prec X \nabla_{b} Y$.

Since we have the initial monosource $\left(h_{a}: X \nabla_{b} Y \rightarrow X \times Y\right)$, it follows that $X \nabla_{b}$ $Y \in \operatorname{EH}(X, Y)$. In other words, every epireflective subcategory of Top containing $X$ and $Y$ must contain $X \nabla_{b} Y$ too. (For the basic properties of $X \nabla_{b} Y$ see [4, Proposition 1]).)

Now we introduce a (sufficient) condition on a space $Y$, under which $X \triangle_{b} Y \in \mathbf{A}$ for each $X \in \mathbf{A}$.

Definition 3.7. Let $Y, Z$ be topological spaces and $b \in Y$ be a point such that the set $\{b\}$ is closed in $Y$. We say that $P(b, Y, Z)$ holds if there exists an open local base $\mathcal{B}$ at $b$ in $Y$, a point $a \in Z$ and an open neighborhood $U_{0}$ of $a$ in $Z$ such that for any $V \in \mathcal{B}$ there exists a continuous map $f: Y \rightarrow Z$ with $f(b)=a, f^{-1}\left(U_{0}\right)=V$. 
We first present some simple examples of spaces with this property. Let $I=$ $\langle 0,1\rangle$. We claim that $P(b, I, I)$ holds for any $b \in I$. We take $\mathcal{B}=\{\langle 0, \varepsilon)\}$ if $b=0$, symmetrically $\mathcal{B}=\{(1-\varepsilon, 1\rangle\}$ if $b=1$, otherwise $\mathcal{B}=\{(b-\varepsilon, b+\varepsilon) \cap I\}$. (For $a$ and $U_{0}$ we can take e.g. 0 and $\left\langle 0, \frac{1}{2}\right), 1$ and $\left(\frac{1}{2}, 1\right\rangle, b$ and $\left(\frac{b}{2}, \frac{1+b}{2}\right)$ respectively.)

Another example: Let $Y$ be any zero-dimensional space, $D_{2}$ be the 2-point discrete space. Any point has a clopen local base, therefore $P\left(b, Y, D_{2}\right)$ holds for any $b \in Y$.

Example 3.8. Let $X$ be any infinite space with the cofinite topology and $b \in X$. Then $P(b, X, X)$.

To show this, we take all neighborhoods of $b$ for $\mathcal{B}$. Let $a=b, u \neq b$ and $U_{0}=X \backslash\{u\}$. Then $U_{0}$ is an open neighborhood of $a$.

Any $V \in \mathcal{B}$ has the form $V=X \backslash F$, where $F$ is a finite set and $b \notin F$. Then there exists a bijection $h: V \rightarrow U_{0}$ such that $h(b)=b$. We can define a map $f: X \rightarrow X$ by $\left.f\right|_{V}=h$ and $f[F]=\{u\}$. Clearly, $f$ is a continuous map.

Lemma 3.9. Let $\mathbf{A}$ be an epireflective subcategory of Top. Let $X, Y, Z \in \mathbf{A}$, $b \in Y$ be a non-isolated point such that $\{b\}$ is closed and $P(b, Y, Z)$ holds. Then $X \triangle_{b} Y \in \mathbf{A}$.

Proof. Using the base $\mathcal{B}$ from the definition of $P(b, Y, Z)$, we can obtain a local base at $(a, b) \in X \triangle_{b} Y$ consisting of sets of the form $\bigcup_{x \in U} V_{x}$, where $U$ is an open neighborhood of $a$ in $X$ and $V_{x} \in \mathcal{B}$ for each $x \in U$. Let $\mathcal{S}=\left\{f_{i}: i \in I\right\}$ be the set of all maps $f_{i}: X \rightarrow \mathcal{B}$. For any basic neighborhood $\bigcup_{x \in U} V_{x}$ there is an $i \in I$ with $f_{i}(x)=V_{x}$ for every $x \in U$. Let us denote the topology of $X \triangle_{b} Y$ by $\mathcal{T}_{\triangle}$.

Now we define the following maps: $p: X \triangle_{b} Y \rightarrow X$ is the projection $p(x, y)=x$, $q: X \triangle_{b} Y \rightarrow X \nabla_{b} Y$ is the identity map $q(x, y)=(x, y)$, and for $i \in I$ we define a map $h_{i}: X \triangle_{b} Y \rightarrow Z$ as follows: For the open neighborhood $f_{i}(x) \in \mathcal{B}$ there exists a continuous map $g_{x, i}: Y \rightarrow Z$ with $g_{x, i}^{-1}\left(U_{0}\right)=f_{i}(x)$ and $g_{x, i}(b)=a$. We put $h_{i}(x, y)=g_{x, i}(y)$. Note that the maps $p, q, h_{i}$ are continuous.

We claim that $\mathcal{T}_{\triangle}$ is the initial topology with respect to the family of maps $\left\{p, q, h_{i} ; i \in I\right\}$. Let us denote this initial topology by $\mathcal{T}$. Let us recall that an open subbase for the initial topology is formed by sets $p^{-1}(U), q^{-1}(U), h_{i}^{-1}(U)=$ $\bigcup_{x \in X} g_{x, i}^{-1}(U)$, where $U$ is any open set in the codomain of the respective map.

$\mathcal{T}_{\triangle} \prec \mathcal{T}$ We first compare open neighborhoods of points $(a, b)$ with $a \in X$. Any such neighborhood contains a basic neighborhood $\bigcup_{x \in U} V_{x}$ with $V_{x} \in \mathcal{B}$ for each $x \in U$. This set can be expressed as $p^{-1}(U) \cap h_{i}^{-1}\left(U_{0}\right)$ for the $i \in I$ satisfying $f_{i}(x)=V_{x}$ for every $x \in U$.

As for neighborhoods of $(a, y), y \neq b$, it suffices to note that they have the same neighborhood bases in $X \triangle_{b} Y$ and $X \nabla_{b} Y$.

$\mathcal{T} \prec \mathcal{T}_{\triangle}$ It suffices to notice that the subbasic sets $p^{-1}(U), q^{-1}(U), h_{i}^{-1}(U)$ belong to $\mathcal{T}_{\triangle}$.

This family of maps forms a monosource, since it contains the identity map. So we have found an initial monosource from $X \triangle_{b} Y$ with a codomain in $\mathbf{A}$ and this implies $X \triangle_{b} Y \in \mathbf{A}$.

Theorem 3.10. Let $\mathbf{A}$ be an epireflective subcategory of Top with $I_{2} \notin \mathbf{A}$ and $\mathbf{B}$ be an HAD-class in $\mathbf{A}$. If $\mathbf{B}$ contains a space $Y$ with $P(b, Y, Z)$ for some $Z \in \mathbf{A}$ and a non-isolated point $b \in Y$ such that the set $\{b\}$ is closed, then $\mathbf{B}$ is closed under prime factors. 
Proof. Follows easily from Proposition 3.3 and Lemma 3.9 .

Corollary 3.11. Let $\mathbf{A}$ be an epireflective subcategory of Top with $I_{2} \notin \mathbf{A}$ and $\mathbf{B}$ be an HAD-class in $\mathbf{A}$. If $\mathbf{B}$ contains an infinite space with the cofinite topology or it contains a non-discrete zero-dimensional space (in particular a prime $T_{2}$-space), then $\mathbf{B}$ is closed under prime factors.

Corollary 3.12. Let $\mathbf{A}$ be an epireflective subcategory of Top with $I_{2} \notin \mathbf{A}$ and $\mathbf{B}$ be an HAD-class in $\mathbf{A}$. If $\mathbf{B}$ contains a prime space, then $\mathbf{B}$ is closed under prime factors.

Proof. If A contains a prime $T_{2}$-space, then the claim follows from Corollary 3.11

Now assume that $\mathbf{A}$ contains a non-Hausdorff prime space $P$. To resolve this case we provide an argument which will be used several more times in this paper.

Since $P$ is not $T_{2}$, there exists a point $b$ which cannot be separated from the non-isolated point $a$ of $P$. The subspace on the set $\{a, b\}$ is homeomorphic to the Sierpiński space $S$. Thus we get $S \in \mathbf{A}, \mathbf{A}=\mathbf{T o p}_{0}$, and the result now follows from Proposition 3.5. (Note that $\mathbf{T o p}_{0}$ is closed under $\triangle$.)

We have seen that the prime $T_{2}$-spaces are more convenient in this context, because they belong to $\mathbf{Z D}_{\mathbf{0}}$ and thus they are automatically contained in any epireflective non-bireflective subcategory. Let us note that in most cases it suffices to consider the prime $T_{2}$-spaces only.

Indeed, the only epireflective subcategory $\mathbf{A}$ of Top with $I_{2} \notin \mathbf{A}$, which contains non- $T_{2}$ prime spaces, is $\mathbf{T o p}_{0}$. Moreover, even for $\mathbf{A}=\mathbf{T o p}_{0}$, the non- $T_{2}$ prime spaces are needed only for AD-classes with $\mathbf{B} \subseteq \mathbf{F G}$.

Using the Corollary 3.11 we can show that if $\mathbf{A} \subseteq$ Haus then every HAD-class $\mathbf{B} \neq$ Disc in A contains a prime space and, consequently, it is closed under the formation of prime factors.

Proposition 3.13. Let $X$ be Hausdorff and not discrete. Then $X$ contains a subspace $Y$, such that there exists a prime $T_{2}$-space $P$ which is a quotient space of $Y$.

Proof. Let $a$ be any non-isolated point in $X$. We would like to get a subspace $Y$ in which $a$ is again non-isolated and which contains enough disjoint open subsets.

By transfinite induction we construct a system $U_{\beta}, \beta<\alpha$, of non-empty open subsets of $X$ such that for each $\beta, \gamma<\alpha$ the following holds:

(1) If $\beta \neq \gamma$ then $U_{\beta} \cap U_{\gamma}=\emptyset$;

(2) $a \in V_{\beta}=X \backslash \overline{\bigcup_{\eta \leq \beta} U_{\eta}}=\operatorname{Int}\left(X \backslash \bigcup_{\eta \leq \beta} U_{\eta}\right)$;

(3) if $\gamma<\beta$ then $U_{\beta} \subseteq V_{\gamma}$;

(4) $a \in \overline{\bigcup_{\eta<\alpha} U_{\eta}}$.

$\beta=0$ : Since $a$ is non-isolated, there exists $b \neq a$ in $X$. By Hausdorffness we have non-empty open sets $U, V$ with $U \cap V=\emptyset, a \in V, b \in U$. We put $U_{0}:=U$. Since $a \in V \subseteq X \backslash U$ and $V$ is open, the condition $a \in V_{0}=\operatorname{Int}\left(X \backslash U_{0}\right)$ is fulfilled. The conditions (1) and (3) are vacuously true in this step of induction.

Now suppose that $U_{\gamma}$ for $\gamma<\beta$ have already been defined. There are two possibilities. Either $a \in \overline{\bigcup_{\eta<\beta} U_{\eta}}$ and we can stop the process (putting $\alpha:=\beta$ ) or $a \notin \overline{\bigcup_{\eta<\beta} U_{\eta}}$. 
In the latter case the set $W:=X \backslash \overline{\bigcup_{<<\beta} U_{\eta}}$ is an open neighborhood of $a$ such that $W \cap\left(\bigcup_{\eta<\beta} U_{\eta}\right)=\emptyset$. Since $a$ is not isolated, there exists $b \in W, b \neq a$. Again, by $T_{2}$-axiom, there exist open sets $U, V$ such that $U \cap V=\emptyset, a \in V, b \in U$. We put $U_{\beta}:=U \cap W$.

Since $U_{\beta} \subseteq W$ and $W \cap\left(\bigcup_{\eta<\beta} U_{\eta}\right)=\emptyset$, we do not violate (1).

The point $a$ belongs to the open set $V \cap W$ and $(V \cap W) \cap U_{\gamma}=\emptyset$ for every $\gamma \leq \beta$, thus we get $a \in \operatorname{Int}\left(X \backslash \bigcup_{\eta \leq \beta} U_{\eta}\right)$, so (2) is fulfilled as well.

For $\gamma<\beta$ we have $W \subseteq V_{\gamma}=X \backslash \overline{\bigcup_{\eta \leq \gamma} U_{\eta}}$, thus $U_{\beta} \subseteq V_{\gamma}$ and (3) holds.

The condition (4) does make sense only at the end of induction, when we have finished the process and said, what $\alpha$ is. Note, that this procedure must stop at some ordinal $\alpha$, otherwise we would obtain a proper class of open subsets of $X$.

Now we put $Y:=\{a\} \cup\left(\bigcup_{\beta<\alpha} U_{\beta}\right)$. The prime space $P$ will be obtained as the space on the set $\alpha \cup\{\alpha\}$ which is quotient with respect to $q: Y \rightarrow P$ defined by $q(a)=\alpha$ and $q\left[U_{\beta}\right]=\{\beta\}$ for any $\beta<\alpha$. By (1) and (2) the map $q$ is well-defined.

Since $q^{-1}(\{\beta\})=U_{\beta}$, each $\beta<\alpha$ is isolated. By (4) and $q^{-1}(\alpha)=\{a\}$, the point $\alpha$ is not isolated. Hence $P$ is a prime space.

Since the set $q^{-1}(\{\gamma \in \alpha \cup\{\alpha\} ; \gamma>\beta\})=V_{\beta} \cap Y$ is open in $Y$ for each $\beta<\alpha$, the prime space $P$ is $T_{2}$ (every isolated point can be separated from the accumulation point).

From Proposition 3.13 and Corollary 3.11 we get

Theorem 3.14. If $\mathbf{A}$ is an epireflective subcategory of Top such that $\mathbf{A} \subseteq$ Haus and $\mathbf{B}$ is an HAD-class in $\mathbf{A}$, then $\mathbf{B}$ is closed under prime factors.

Corollary 3.15. Let $\mathbf{A}$ be an epireflective subcategory of Top such that $\mathbf{A} \subseteq$ Haus. For every HAD-class $\mathbf{B}$ in $\mathbf{A}$ there exists a class $\mathbf{S}$ of prime spaces such that $\mathbf{B}=$ $\mathrm{AD}_{\mathbf{A}}(\mathbf{S})$.

3.2. Two related examples. In the rest of this section we present two examples which are connected with HAD-classes and hereditary coreflective subcategories. In the first one we will deal with closedness of epireflective subcategories under $\triangle$. The second one is an example of an AD-class in Tych which is not coreflective in Tych.

We have observed in Proposition 3.5 that if $\mathbf{A}$ is closed under $\triangle$ then every HAD-class in $\mathbf{A}$ is closed under prime factors. We also noticed that this condition is fulfilled for many familiar epireflective subcategories of Top. Now we provide an example showing that it does not hold in general.

We first recall the notion of a strongly rigid space. A topological space $X$ is called strongly rigid if any continuous map $f: X \rightarrow X$ is either constant or $i d_{X}$. (See [17, it should be noted that such spaces are called rigid by some authors.) We will show in Example 3.17 that for a strongly rigid space which is not "too trivial" $X \triangle_{b} X \in \mathrm{EH}(X)$ does not hold.

Lemma 3.16. Let $X$ be a topological space and $b \in X$. If $X$ is a strongly rigid space and $X \triangle_{b} X \in \mathrm{EH}(X)$, then for $x \neq b$ the set $\{U \times V ; U$ is an open neighborhood of $x$ and $V$ is an open neighborhood of $b\}$ is a local base for the topology of $X \triangle_{b} X$ 
at $(x, b)$, i.e., this topology has the same local base at the point $(x, b)$ as the product topology.

Proof. Recall, that the underlying set of $X \triangle_{b} X$ is $X \times X$ (see Definition 3.2). Clearly, $X \triangle_{b} X \prec X \times X$. So it remains to show that any neighborhood of $(x, b)$ in $X \triangle_{b} X$ contains a neighborhood of the form $U \times V$ with $U$ and $V$ as above. Since we assume that $X \triangle_{b} X \in \mathrm{EH}(X)$, the space $X \triangle_{b} X$ has the initial topology w.r.t. the family $C\left(X \triangle_{b} X, X\right)$; i.e., the subbase for the topology of this space consists of sets $f^{-1}(U)$ where $f \in C\left(X \triangle_{b} X, X\right)$ and $U$ is open subset of $X$. (By $C(Y, Z)$ we mean the family of all continuous mapping between spaces $Y$ and $Z$.)

The subspaces of $X \triangle_{b} X$ on the sets $\{a\} \times X$ for any $a \in X$ and $X \times\{b\}$ are homeomorphic to $X$. To be more precise, the homeomorphisms are given by $h_{a}(a, x)=x$ (between the subspace $\{a\} \times X$ and $\left.X\right)$ and $h(x, b)=x$ (between the subspace $X \times\{b\}$ and $X)$. Thus for any $f \in C\left(X \triangle_{b} X, X\right)$ the restrictions to these subspaces are either constant or coincidental with $h_{a}$ resp. $h$. We next investigate in detail all maps $f$ in $C\left(X \triangle_{b} X, X\right)$.

First, assume that $\left.f\right|_{X \times\{b\}}$ is not constant. Then $f(x, b)=x$ for any $x \in X$. Thus for $a \neq b$ we get $f(a, b)=a \neq b=h_{a}(a, b)$. Therefore the restriction of $f$ to the subspace $\{a\} \times X, a \neq b$, is the constant map $f(a, x)=a$. For the subspace $\{b\} \times X$ we have two possibilities: $h_{b}$ or a constant map. In this case we obtain two continuous maps: $f_{1}$ such that $f_{1}(x, y)=x$ and $f_{2}$ given by $f_{2}(x, y)=x$ for $x \neq b$ and $f_{2}(b, y)=y$.

The second possibility remains: $f(x, b)=a_{0}$ for any $x \in X$. If $a_{0} \neq b$ then for any $a \in X$ we have $f(a, b)=a_{0} \neq b=h_{a}(a, b)$ and $f$ is a constant map. Thus the only interesting case is $a_{0}=b$. In this case some restrictions are equal to $h_{a}$ 's and some are constant. I.e., every such map corresponds to a subset $A$ of $X$ in the following way: $f_{A}(x, y)=y$ if $x \in A$ and $f_{A}(x, y)=b$ otherwise.

We showed that the family $C\left(X \triangle_{b} X, X\right)$ consists precisely of all constant maps, the maps $f_{1}, f_{2}$ and the maps of the form $f_{A}, A \subseteq X$. The set $U \times V$ can be obtained as $f_{X}^{-1}(V) \cap f_{1}^{-1}(U)$. Moreover, every subbasic set $f^{-1}(U)$, where $f \in C\left(X \triangle_{b} X, X\right)$, contains a subset of the form $U \times V$. Thus such sets from a local base.

Example 3.17. If $X$ is a strongly rigid space and $\bigcap_{x \in X} U_{x}$ is an intersection of open neighborhoods of a point $b$ in $X$ which fails to be a neighborhood, then the set $\bigcup_{x \in X}\{x\} \times U_{x}$ is open in $X \triangle_{b} X$, but it does not contains any subset from the local basis described in Lemma 3.16. Therefore in such case $X \triangle_{b} X \notin \operatorname{EH}(X)$.

This means that to obtain a counterexample, it suffices to have a strongly rigid space with a non-isolated point $b$ such that at the same time $\{b\}$ is an intersection of a family $U_{i}, i \in I$, of open sets, with card $I \leq \operatorname{card} X$. Any of the examples of strongly rigid $T_{2}$-spaces constructed in [5], 8] or [17] satisfies this condition.

We next include an example of an AD-class which is not coreflective. We will work in the epireflective subcategory $\mathbf{A}=\mathbf{T y c h}$ of all completely regular (Tychonoff) spaces.

A topological space $X$ is called $k_{R}$-space if it is completely regular and if every map $f: X \rightarrow \mathbb{R}$, whose restriction to every compact subset $K \subseteq X$ is continuous, is continuous on $X$. For more information about $k_{R}$-spaces see e.g. [13] or [18].

A topological space $X$ is a $k$-space if a subset $U \subseteq X$ is open whenever $U \cap$ $K$ is open for every compact subset $K \subseteq X$. The class of all $k$-spaces is the 
coreflective hull of compact spaces in Top. In the next example we will denote the corresponding coreflector by $C$. It is known that $X$ and $C X$ have precisely the same compact subsets and the relative topology on every compact subset is the same.

Example 3.18. E. Michael constructed in [20, Lemma 3.8] a normal $k_{R}$-space $X$ such that $C X$ is not regular. This means that $X$ is not a $k$-space. We will show that $R C X=X$, where $R$ denotes the Tych-reflection. This implies that $X$ is in the coreflective hull of compact spaces in Tych, since there exists a quotient map $q$ from the sum of all compact subspaces of $X$ to $C X$ and consequently the map $R q$ with the codomain $R C X=X$ is a Tych-extremal epimorphism. (Since every reflector is coadjoint functor, it preserves regular epimorphisms.) But $X$ is not in the AD-hull of compact spaces in Tych, since $C X \neq X$. Therefore the AD-hull of compact spaces in Tych (which consists precisely of Hausdorff $k$-spaces) is an example of an AD-class in Tych which is not a coreflective subcategory of Tych.

To show that $X$ is the Tych-reflection of $C X$ it suffices to show that the continuous maps from both spaces to $\mathbb{R}$ are the same. A map $f: C X \rightarrow \mathbb{R}$ is continuous if and only if all restrictions $\left.f\right|_{K}$ with $K$ compact are continuous. Since compact subsets of $X$ and $C X$ are the same and moreover the corresponding subspaces are homeomorphic, this implies that $f$ is continuous as the map from $X$ to $\mathbb{R}$. (Since $X$ is a $k_{R}$-space and we have shown that the restrictions on compact subsets are continuous.)

\section{The SPACE $A_{\omega}$ AND HAD-Classes}

In 23] the space $A_{\omega}$ was constructed for any prime space $A$ and it was shown that the prime factor $\left(A_{\omega}\right)_{a}$ is a generator of the hereditary coreflective hull of the space $A$ in Top. The goal of this section is to show some useful properties of the space $A_{\omega}$. Namely we will prove that this space is zero-dimensional for any prime $T_{2}$-space $A$. This implies that, if $A$ is a prime $T_{2}$-space, then $A_{\omega}$ is contained in every epireflective subcategory $\mathbf{A}$ with $I_{2} \notin \mathbf{A}$. Using this property of $A_{\omega}$ we can show that if $\mathbf{B}$ is an HAD-class in $\mathbf{A}$ which contains a prime space then the coreflective hull $\mathrm{CH}(\mathbf{B})$ of $\mathbf{B}$ in Top is hereditary.

We first introduce some notions which are necessary to define the space $A_{\omega}$. This space is very similar to the space $S_{\omega}$ defined in [2], the difference lies in using an arbitrary prime space $A$ instead of the space $C(\omega)$ (see Definition 6.1) and $A$-sums instead of sequential sums. Let us note that the space $S_{\omega}$ is also a special case of the space $\mathcal{T}_{\overrightarrow{\mathcal{F}}}$ defined in [24].

We first recall the definition of $A$-sums from [23]. Apart from the sequential sums of [2] this construction is also similar to the brush of [16].

Definition 4.1. Let $A$ be a prime space with the accumulation point $a$. Let us denote $B:=A \backslash\{a\}$. Suppose that for each $b \in B$ we are given a topological space $X_{b}$ and a point $x_{b} \in X_{b}$. Then the $A$-sum $\sum_{A}\left\langle X_{b}, x_{b}\right\rangle$ is the topological space on the set $F=A \cup\left(\bigcup_{b \in B}\{b\} \times\left(X_{b} \backslash\left\{x_{b}\right\}\right)\right)$ which is quotient with respect to the map $\varphi: A \sqcup\left(\coprod_{b \in B} X_{b}\right) \rightarrow F, \varphi(x)=x$ for $x \in A, \varphi(x)=(b, x)$ for $x \in X_{b} \backslash\left\{x_{b}\right\}$ and $\varphi\left(x_{b}\right)=b$ for every $b \in B$.

This means that the $A$-sum is defined simply by identifying each $x_{b} \in X_{b}$ with the corresponding point $b \in A$. 
For the sake of convenience, we adopt some terminology from [16]. The map $\varphi$ is called the defining map of the $A$-sum. The subspace of $\sum\left\langle X_{b}, x_{b}\right\rangle$ on the subset $\varphi\left[X_{b}\right]=\{b\} \cup\left(\{b\} \times\left\{X_{b} \backslash\left\{x_{b}\right\}\right\}\right)$ is called the bristle.

The following easy lemma states that the bristles are homeomorphic to the spaces from which the $A$-sum is constructed.

Lemma 4.2. The space $X_{b}$ is homeomorphic to the subspace of the space $\sum\left\langle X_{b}, x_{b}\right\rangle$ on the subset $\varphi\left[X_{b}\right]=\{b\} \cup\left(\{b\} \times\left\{X_{b} \backslash\left\{x_{b}\right\}\right\}\right)$. (The homeomorphism is given by the restriction of $\varphi$ to the summand $X_{b}$.)

Now we are ready to define the space $A_{\omega}$ using the $A$-sum. We first define inductively the spaces $A_{n}$ for $n \in \mathbb{N}$. We put $A_{1}=A$ and $A_{n+1}=\sum\left\langle A_{n}, a\right\rangle$. Note that $A_{n}$ is a subspace of $A_{n+1}$ for each $n$. Figure 3 depicts the space $A_{3}$ for $A=C(\omega)$.

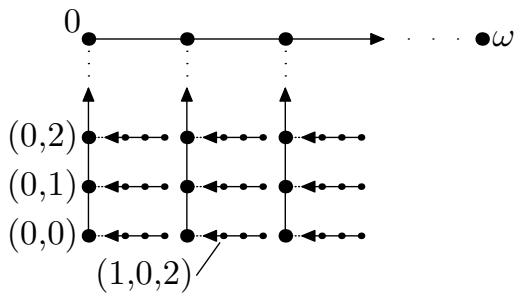

Figure 3 . The space $A_{3}$ for $A=C(\omega)$

Another possibility how to obtain $A_{n+1}$ is to attach the space $A$ (by its accumulation point) to each isolated point of $A_{n}$. Clearly, the underlying set is the same. It can be shown by induction that the topologies are the same too.

For $n=1$ this is clear from the definition of $A_{2}$. If $n>1$ then each stem of the space $A_{n+1}$ is homeomorphic to $A_{n}$. By the induction hypothesis it can be obtained by gluing the space $A$ to each isolated point of $A_{n-1}$. The isolated points in $A_{n}=\sum A_{n-1}$ are precisely the isolated points of the bristles. Hence by attaching the space $A$ to isolated points we get $A_{n}$ from each bristle and the resulting space will be $A_{n+1}=\sum A_{n}$.

Definition 4.3. Let $A$ be a prime space with the accumulation point $a$. Then $A_{\omega}$ is the space on the set $\bigcup_{n \in \mathbb{N}} A_{n}$ where $U \subseteq \bigcup_{n \in \mathbb{N}} A_{n}$ is open if and only if $U \cap A_{n}$ is open for every $n \in \mathbb{N}$.

We see that $A_{\omega}$ is a quotient space of $\coprod_{n \in \mathbb{N}} A_{n}$, so $A_{\omega} \in \mathrm{CH}(A)$. (Let us note that $A_{\omega}$ is the inductive limit of spaces $A_{n}$ and each $A_{n}$ is embedded into $A_{\omega}$.)

Observe that the underlying set of the space $A_{\omega}$ is $\{a\} \cup \bigcup_{n \in \mathbb{N}}(A \backslash\{a\})^{n}$. The space $A_{n}$ is homeomorphic to the subspace on the subset $\{a\} \cup \bigcup_{k=1}^{n}(A \backslash\{a\})^{k}$ and the bristles of $A_{n}$ are the subspaces on sets $\{b\} \cup\left\{\left(b, x_{2}, x_{3}, \ldots, x_{n}\right) ; x_{i} \in A \backslash\{a\}\right\}$.

We introduce some terminology analogous to [2. We say that a point $x \in A_{\omega}$ is a point of $k$-th level if it belongs to $(A \backslash\{a\})^{k}$. Point $a$ is the only point of 0 -th level.

It was proved in [23, Proposition 5.1] that $A_{\omega}$ homeomorphic to the $A$-sum of several copies of itself: $A_{\omega} \cong \sum_{A}\left\langle A_{\omega}, a\right\rangle$. 
From this property and Lemma 4.2 we can see that the bristles are homeomorphic to $A_{\omega}$.

Lemma 4.4. For $b \in A \backslash\{a\}$ denote by $X$ the subspace of $A_{\omega}$ on the set $X:=$ $\{b\} \cup\left(\{b\} \times \bigcup_{n \in \mathbb{N}}(A \backslash\{a\})^{n}\right)$. Then $X$ is homeomorphic to $A_{\omega}$. Moreover, if $A$ is $T_{2}$, then $X$ is a clopen subset of $A_{\omega}$.

Using this lemma repeatedly we can find for any point of $A_{\omega}$ a (clopen, if $A$ is $T_{2}$ ) neighborhood homeomorphic to $A_{\omega}$ (by induction on the level of points). Namely, if $x=\left(x_{1}, \ldots, x_{k}\right)$ then $U_{x}=\left\{\left(x_{1}, \ldots, x_{k}\right)\right\} \cup\left\{\left(x_{1}, \ldots, x_{k}\right)\right\} \times\left(\bigcup_{n \in \mathbb{N}}(A \backslash\{a\})^{n}\right)$ is a neighborhood of $x$ homeomorphic to $A_{\omega}$.

We now proceed to defining a clopen local base at $a \in A_{\omega}$.

We will show that $\mathcal{B}=\left\{U \subseteq A_{\omega} ; a \in U ; U\right.$ is open in $A_{\omega}$ and (11) holds $\}$ is a base for $A_{\omega}$ at the point $a$.

$$
\left(x_{1}, x_{2}, \ldots, x_{n}, x_{n+1}, \ldots, x_{n+k}\right) \in U \Rightarrow\left(x_{1}, x_{2}, \ldots, x_{n}\right) \in U
$$

Lemma 4.5. $\mathcal{B}$ is a base for $A_{\omega}$ at the point a.

Proof. Let $V$ be an open neighborhood of $a$. We want to find $U \in \mathcal{B}$ such that $U \subseteq V$. Let us put

$$
\begin{aligned}
U_{1} & :=V \cap A_{1} \\
U_{2} & :=V \cap A_{2} \cap\left[U_{1} \cup\left(U_{1} \times(A \backslash\{a\})\right)\right] \\
U_{n+1} & :=V \cap A_{n+1} \cap\left[U_{n} \cup\left(U_{n} \times(A \backslash\{a\})\right)\right]
\end{aligned}
$$

and $U:=\bigcup_{n \in \mathbb{N}} U_{n}$.

Observe that $a \in U \subseteq V$ and, for each $n \in \mathbb{N}, U_{n} \subseteq U_{n+1}, U_{n}$ is open in $A_{n}$ and $U \cap A_{n}=U_{n}$. Hence $U$ is open in $A_{\omega}$.

If $\left(x_{1}, \ldots, x_{n+1}\right) \in U_{n+1}$ then $\left(x_{1}, \ldots, x_{n+1}\right) \in U_{n} \times(A \backslash\{a\})$ and $\left(x_{1}, \ldots, x_{n}\right) \in$ $U_{n}$. By induction we get that (1) holds for $U$.

Lemma 4.6. All sets in $\mathcal{B}$ are clopen.

Proof. Let $U$ be any set from $\mathcal{B}$. If $x=\left(x_{1}, \ldots, x_{k}\right) \notin U$, then no point of the form $\left(x_{1}, \ldots, x_{k}, y_{k+1}, \ldots, y_{k+l}\right)$ belongs to $U$, i.e., $U_{x} \cap U=\emptyset$ holds for the neighborhood $U_{x}=\left\{\left(x_{1}, \ldots, x_{k}\right)\right\} \cup\left\{\left(x_{1}, \ldots, x_{k}\right)\right\} \times\left(\bigcup_{n \in \mathbb{N}}(A \backslash\{a\})^{n}\right)$ of $x$. Hence $x \in \operatorname{Int}\left(A_{\omega} \backslash U\right)$, $A_{\omega} \backslash U$ is open, $U$ is closed.

If $A$ is $T_{2}$ then by Lemma 4.4 we obtain from the clopen base $\mathcal{B}$ at $a$ a clopen base at each point of $A_{\omega}$. Thus we get finally

Proposition 4.7. The space $A_{\omega}$ is zero-dimensional and $T_{2}$ for any prime $T_{2}$-space $A$.

As $A_{\omega}$ is a zero-dimensional $T_{2}$-space, it is contained in any epireflective subcategory of Top with $I_{2} \notin \mathbf{A}$. In the following proposition we summarize some properties of $A_{\omega}$ which were proved in [23].

Proposition 4.8. If $A$ is a prime space, then $\mathrm{HCH}(A)=\mathrm{CH}\left(\left(A_{\omega}\right)_{a}\right)=\mathrm{HCH}\left(A_{\omega}\right)$.

If $A$ is infinite then $A_{\omega} \in \mathrm{CH}(A)$ and $\operatorname{card}\left(A_{\omega}\right)_{a}=\operatorname{card} A$.

Using Lemma 2.1 we obtain from Proposition 4.8 the following corollary.

Corollary 4.9. Let $\mathbf{A}$ be an epireflective subcategory of Top with $I_{2} \notin \mathbf{A}$. If $A \in \mathbf{A}$ is a prime space, then $\operatorname{HAD}_{\mathbf{A}}(A)=\mathrm{AD}_{\mathbf{A}}\left(\left(A_{\omega}\right)_{a}\right)=\mathrm{HCH}(A) \cap \mathbf{A}$. Moreover, if $A$ is infinite, then $\operatorname{card}\left(A_{\omega}\right)_{a}=\operatorname{card} A$. 
Proof. We first observe that $\mathbf{A}$ contains $A_{\omega}$. If $A$ is $T_{2}$ then this is true by Proposition 4.7 If $A$ is not $T_{2}$, then $\mathbf{A}=\mathbf{T o p}_{0}$ and $A_{\omega}$ is clearly a $T_{0}$-space.

By Lemma 2.1 and Proposition $4.8 \operatorname{HAD}_{\mathbf{A}}(A) \subseteq \operatorname{HCH}(A) \cap \mathbf{A}=\mathrm{CH}\left(\left(A_{\omega}\right)_{a}\right) \cap$ $\mathbf{A}=\operatorname{AD}_{\mathbf{A}}\left(\left(A_{\omega}\right)_{a}\right)$ holds.

On the other hand, $A_{\omega} \in \mathbf{A}$ implies $A_{\omega} \in \operatorname{HAD}_{\mathbf{A}}(A)$. The HAD-class $\operatorname{HAD}_{\mathbf{A}}(A)$ contains the prime space $A$. So by Corollary 3.12 it is closed under prime factors and $\left(A_{\omega}\right)_{a} \in \operatorname{HAD}_{\mathbf{A}}(A)$, which proves the opposite inclusion.

In the rest of this section we show that coreflective hull $\mathrm{CH}(\mathbf{B})$ in Top of an HAD-class $\mathbf{B}$ in $\mathbf{A}$ is hereditary whenever $\mathbf{B}$ contains at least one prime space.

Lemma 4.10. Let $\mathbf{A}$ be an epireflective subcategory of Top such that $I_{2} \notin \mathbf{A}$. If $\mathbf{B}=\operatorname{HAD}_{\mathbf{A}}(\mathbf{D})$, where $\mathbf{D} \subseteq \mathbf{A}$ is a set of spaces and $\mathbf{B}$ contains at least one prime space, then there exists a prime space $B \in \mathbf{A}$ such that $\mathbf{B}=\operatorname{HAD}_{\mathbf{A}}(B)=\operatorname{AD}_{\mathbf{A}}(B)$. Moreover, $\mathrm{CH}(B)=\mathrm{HCH}(B)$ is hereditary.

Proof. Let us denote by $\mathbf{D}^{\prime}$ the set of all non-discrete prime factors of spaces from D. By joining the accumulation points of all prime spaces in $\mathbf{D}^{\prime}$ into one point we get a prime space $A$. We consider 2 cases. If $\mathbf{A}=\mathbf{T o p}_{0}$ then clearly $A \in \mathbf{A}$. If $\mathbf{A} \subseteq \mathbf{T o p}_{1}$ then all spaces in $\mathbf{D}^{\prime}$ are $T_{2}$ and $A$ is $T_{2}$ as well. Therefore in both cases $A \in \mathbf{A}$ and $\operatorname{HAD}_{\mathbf{A}}(A)=\operatorname{HAD}_{\mathbf{A}}\left(\mathbf{D}^{\prime}\right)$.

Any space from $\mathbf{D}$ can be obtained as a quotient of the sum of its prime factors and consequently $\mathrm{AD}_{\mathbf{A}}\left(\mathbf{D}^{\prime}\right)=\mathrm{CH}\left(\mathbf{D}^{\prime}\right) \cap \mathbf{A}$ contains the whole $\mathbf{D}$ and $\mathbf{B}=\operatorname{HAD}_{\mathbf{A}}(\mathbf{D})=\operatorname{HAD}_{\mathbf{A}}\left(\mathbf{D}^{\prime}\right)=\operatorname{HAD}_{\mathbf{A}}(A)$.

Using Corollary 4.9 we obtain that the claim of the lemma holds for $B=\left(A_{\omega}\right)_{a}$.

With the help of Lemma 4.10 we can prove, using very similar methods as in 4, Proposition 4], the following theorem.

Theorem 4.11. Let $\mathbf{A}$ be an epireflective subcategory of Top such that $I_{2} \notin \mathbf{A}$. If $\mathbf{B}$ is an HAD-class in $\mathbf{A}$ and $\mathbf{B}$ contains at least one prime space, then the coreflective hull $\mathrm{CH}(\mathbf{B})$ of $\mathbf{B}$ in Top is hereditary.

Proof. We first represent $\mathbf{B}$ as a union of an ascending chain of HAD-classes $\mathbf{B}_{\alpha}$ in $\mathbf{A}$, such that each of them is generated by a single space (as an AD-class).

Let us denote by $\mathbf{B}_{\alpha}$ the HAD-hull of all spaces from $\mathbf{B}$ with cardinality at most $\alpha$. Clearly, $\mathbf{B}=\bigcup_{\alpha \in \mathrm{Cn}} \mathbf{B}_{\alpha}$ and the system $\mathbf{B}_{\alpha}, \alpha \in \mathrm{Cn}$, is nondecreasing.

Since $\mathbf{B}$ contains a prime space, there exists the smallest $\alpha_{0}$ such that $\mathbf{B}_{\alpha_{0}}$ contains a prime space. Then $\mathbf{B}=\bigcup_{\alpha \geq \alpha_{0}} \mathbf{B}_{\alpha}$ and for each $\alpha \geq \alpha_{0}$ the class $\mathbf{B}_{\alpha}$ is a HAD-hull of a set of spaces and it contains a prime space. So we can use Lemma 4.10 and we get that for any cardinal number $\alpha \geq \alpha_{0}$ there exists a prime space $B_{\alpha} \in \mathbf{A}$ such that $\mathbf{B}_{\alpha}=\operatorname{HAD}_{\mathbf{A}}\left(B_{\alpha}\right)=\operatorname{AD}_{\mathbf{A}}\left(B_{\alpha}\right) \subseteq \mathrm{CH}\left(B_{\alpha}\right)$.

It is easy to see that $\mathrm{CH}(\mathbf{B})$ consists of quotients of spaces from $\mathbf{B}$. Thus if $Y \in \mathrm{CH}(\mathbf{B})$, then $Y$ is quotient of some space $X \in \mathbf{B}$ and there exists $\alpha \geq \alpha_{0}$ such that $X \in \mathbf{B}_{\alpha}$. Consequently we get $Y \in \mathrm{CH}\left(B_{\alpha}\right)$.

Any subspace of $Y$ belongs to $\mathrm{HCH}\left(B_{\alpha}\right)=\mathrm{CH}\left(B_{\alpha}\right) \subseteq \mathrm{CH}(\mathbf{B})$. Thus $\mathrm{CH}(\mathbf{B})$ is closed under the formation of subspaces. $1]$.

Using the above theorem we can prove the result corresponding to [4, Corollary 
Corollary 4.12. Let $\mathbf{A}$ be an epireflective subcategory of Top such that $I_{2} \notin \mathbf{A}$. Let $\mathbf{B} \subseteq \mathbf{A}$ and $\mathbf{B}$ contain at least one prime space. Then $\operatorname{HAD}_{\mathbf{A}}(\mathbf{B})=\mathrm{HCH}(\mathbf{B}) \cap \mathbf{A}=$ $\mathrm{S}(\mathrm{CH}(\mathbf{B})) \cap \mathbf{A}$.

Proof. By Lemma 2.1 we have $\operatorname{HAD}_{\mathbf{A}}(\mathbf{B}) \subseteq \mathrm{HCH}(\mathbf{B}) \cap \mathbf{A}$.

To obtain the opposite inclusion, we use Theorem 4.11 for the HAD-class $\operatorname{HAD}_{\mathbf{A}}(\mathbf{B})$. We get $\mathrm{CH}\left(\mathrm{HAD}_{\mathbf{A}}(\mathbf{B})\right)=\mathrm{HCH}\left(\mathrm{HAD}_{\mathbf{A}}(\mathbf{B})\right)$. This implies $\operatorname{HAD}_{\mathbf{A}}(\mathbf{B}) \supseteq \mathrm{CH}\left(\operatorname{HAD}_{\mathbf{A}}(\mathbf{B})\right) \cap$ $\mathbf{A}=\operatorname{HCH}\left(\operatorname{HAD}_{\mathbf{A}}(\mathbf{B})\right) \cap \mathbf{A} \supseteq \operatorname{HCH}(\mathbf{B}) \cap \mathbf{A}$.

In particular, Theorem 3.14 implies that Theorem 4.11 and Corollary 4.12 are valid for any HAD-class in $\mathbf{A} \subseteq$ Haus.

Corollary 4.13. For any epireflective subcategory $\mathbf{A}$ of Top such that $\mathbf{A} \subseteq$ Haus the assignment given by $\mathbf{C} \mapsto \mathbf{C} \cap \mathbf{A}$ yields a bijection between the hereditary coreflective subcategories of Top with $\mathbf{C} \supseteq \mathbf{F G}$ and HAD-classes in $\mathbf{A}$.

Proof. If $\mathbf{C}$ is a hereditary coreflective subcategory of Top then the class $\mathbf{C} \cap \mathbf{A}$ is an intersection of two hereditary classes, thus it is hereditary as well. It is clearly an AD-class in $\mathbf{A}$.

Let us denote by $F$ the assignment defined in the claim. We will show that $G$ given by $G(\mathbf{D i s c})=\mathbf{F G}$ and $G(\mathbf{B})=\mathrm{CH}(\mathbf{B})$ for $\mathbf{B} \neq \mathbf{D i s c}$ is inverse to $F$.

First, observe that if $\mathbf{B} \neq \mathbf{D i s c}$ is an HAD-class in $\mathbf{A}$ then by Proposition 3.13 it contains a prime space and from Theorem 4.11 we get that $\mathrm{CH}(\mathbf{B})$ is hereditary.

Let $\mathbf{C} \supsetneq \mathbf{F G}$ be a hereditary coreflective subcategory of Top. Then $G(F(\mathbf{C}))=$ $\mathrm{CH}(\mathbf{C} \cap \mathbf{A})$. Since $\mathbf{C} \cap \mathbf{A}$ contains all prime $T_{2}$-spaces from $\mathbf{C}$, we get $\mathrm{CH}(\mathbf{C} \cap \mathbf{A})=$ $\mathbf{C}$ (see 4, Lemma 1]). The equality $G(F(\mathbf{F G}))=\mathbf{F G}$ is also clear.

On the other hand, if $\mathbf{B}$ is an HAD-class in $\mathbf{A}$ and $\mathbf{B} \neq \mathbf{D i s c}$, then $F(G(\mathbf{B}))=$ $\mathrm{CH}(\mathbf{B}) \cap \mathbf{A}=\mathrm{AD}_{\mathbf{A}}(\mathbf{B})=\mathbf{B}$.

Remark 4.14. Obviously, if $\mathbf{A}$ is an epireflective subcategory of Top with $\mathbf{A} \subseteq$ Haus (e.g. $\mathbf{A}=\mathbf{T y c h}, \boldsymbol{R e g}_{2}, \mathbf{Z D}_{\mathbf{0}}$ ), then $\mathbf{C} \mapsto \mathbf{C} \cap \mathbf{A}$ yields a bijection between the hereditary coreflective subcategories (i.e., HAD-classes) in Haus and HAD-classes in $\mathbf{A}$.

For any epireflective subcategory of Top with $I_{2} \notin \mathbf{A}$ the above assignment is a bijection between hereditary coreflective subcategories of Top such that $\mathbf{C} \supsetneq$ FG and the HAD-classes in $\mathbf{A}$ containing at least one prime space.

\section{Extension of the RESUlts to BIREFlective SUbCATEGORIES}

Until now we have only dealt with the epireflective subcategories $\mathbf{A}$ of Top such that $I_{2} \notin \mathbf{A}$, i.e., with $\mathbf{A}$ not bireflective. In this section we would like to find a method how to extend our results also to bireflective subcategories of Top. For this, we can use the one-to-one correspondence between bireflective and nonbireflective epireflective subcategories of Top given by the assignments $\mathbf{A} \mapsto \mathrm{BH}(\mathbf{A})$ and $\mathbf{B} \mapsto \mathbf{B} \cap \mathbf{T o p}_{0}=\left\{R_{0} B ; B \in \mathbf{B}\right\}$ (see [19], [21]). For sake of simplicity we will ignore the trivial case $\mathbf{A}=$ Ind. (The bireflective subcategory Ind corresponds in this assignment to the subcategory containing only one-point spaces and the empty space.)

Recall that the category Top $_{0}$ of all $T_{0}$-spaces is a quotient-reflective subcategory of Top. We will denote the $T_{0}$-reflector by $R_{0}$.

The $T_{0}$-reflection of a space $X$ is the quotient space given by the following equivalence relation: $x \sim y$ if and only if $\overline{\{x\}}=\overline{\{y\}}$ (see e.g. [9, Beispiel 8.3(2)]). The 
$T_{0}$-reflection arrow is the quotient map corresponding to this equivalence relation. It is moreover an initial map and a retraction, i.e., the $T_{0}$-reflection $R_{0} X$ is homeomorphic to a subspace of $X$ obtained by choosing one point from each equivalence class.

Using the results of the foregoing section we obtain a simple characterization of hereditary AD-classes in a bireflective subcategory $\mathbf{A}$ in Theorem 5.4.

The following lemma says that in the collection of all AD-classes in $\mathbf{A}$ with $I_{2} \in \mathbf{A}$ all AD-classes except Disc contain $I_{2}$.

Lemma 5.1. Let $\mathbf{C}$ be an $A D$-class in an epireflective subcategory $\mathbf{A}$ with $I_{2} \in \mathbf{A}$ and $\mathbf{A} \neq \mathbf{I n d}$. If $\mathbf{C}$ contains a non-discrete space then $I_{2} \in \mathbf{C}$.

Proof. Let $C \in \mathbf{C}$ be a non-discrete space and $c \in C$ be non-isolated. Let us define $f, g: C \rightarrow I_{2}$ by $f(c)=0, f[C \backslash\{c\}]=\{1\}$ and $g(c)=1, g[C \backslash\{c\}]=\{0\}$. We see at once that the map $h: C \sqcup C \rightarrow I_{2}$, obtained as the combination of $f$ and $g$, is a quotient map.

Lemma 5.2. Let $\mathbf{C}$ be an $A D$-class in an epireflective subcategory $\mathbf{A}, \mathbf{A} \neq \mathbf{I n d}$, $I_{2} \in \mathbf{C}$ and $A \in \mathbf{A}$. Then $A \in \mathbf{C}$ if and only if $R_{0} A \in \mathbf{C}$.

Proof. Let $A \in \mathbf{C}$. Since $R_{0} A$ is a subspace of $A$, we have $R_{0} A \in \mathbf{A}$. The $T_{0^{-}}$ reflection arrow $A \rightarrow R_{0} A$ is a quotient map, therefore $R_{0} A \in \mathbf{C}$.

Now let $R_{0} A \in \mathbf{C}$. Since the $T_{0}$-reflection arrow $r_{A}: A \rightarrow R_{0} A$ is an initial map and bireflective subcategories of Top are known to be closed under initial sources, we get $A \in \mathrm{BH}\left(\mathrm{R}_{0} \mathrm{~A}\right)=\mathrm{EH}\left(\left\{R_{0} A, I_{2}\right\}\right) \subseteq \mathbf{A}$.

Since the equivalence classes of $\sim$ are indiscrete subspaces of $A$, the space $A$ can be obtained as a quotient of the topological sum of $R_{0} A$ and indiscrete spaces corresponding to these equivalence classes. (We identify each point of $R_{0} A$ with some point from the indiscrete space representing its equivalence class.) Hence $A \in \mathrm{CH}\left(R_{0} A, I_{2}\right)$. Consequently, $A \in \mathbf{C}$.

Lemma 5.3. If $\mathbf{C}$ is an $A D$-class in an epireflective subcategory $\mathbf{A} \neq \mathbf{I n d}$, then $\mathbf{C} \cap \mathbf{T o p}_{0}=R_{0} \mathbf{C}=\left\{R_{0} C ; C \in \mathbf{C}\right\}$.

Proof. W.l.o.g. let $\mathbf{C}$ contains a non-discrete space.

If $X \in \mathbf{C} \cap \mathbf{T o p}_{0}$, then $X=R_{0} X \in R_{0} \mathbf{C}$. Hence $\mathbf{C} \cap \mathbf{T o p}_{0} \subseteq R_{0} \mathbf{C}$.

On the other hand, let $X=R_{0} C$ for $C \in \mathbf{C}$. By Lemma $5.2 X \in \mathbf{C}$, hence $X \in \mathbf{C} \cap \mathbf{T o p}_{0}$. So the opposite inclusion is true as well.

Theorem 5.4. Let $\mathbf{C}$ be an AD-class in an epireflective subcategory $\mathbf{A} \neq \mathbf{I n d}$ with $I_{2} \in \mathbf{A}$. Then $\mathbf{C}$ is hereditary if and only if $R_{0} \mathbf{C}=\mathbf{C} \cap \mathbf{T o p}_{0}$ is hereditary.

Proof. We can assume $I_{2} \in \mathbf{C}$, since otherwise $\mathbf{C} \subseteq$ Disc and the claim is trivial.

If $\mathbf{C}$ is hereditary, then $\mathbf{C} \cap \mathbf{T o p}{ }_{0}$ is hereditary as an intersection of two hereditary classes.

Now assume that $R_{0} \mathbf{C}$ is hereditary. Let $B \in \mathbf{C}$ and $e: A \hookrightarrow B$ be an embedding. Then the map $R_{0} e: R_{0} A \hookrightarrow R_{0} B$ is an embedding as well. (Recall that $R_{0} B$ is the subspace of $B$ obtained by choosing one point from each equivalence class and note that the equivalence relation $\sim_{A}$ is the restriction of the relation $\sim_{B}$.) Therefore $R_{0} A \in \mathbf{C} \cap \mathbf{T o p}_{0}$. Then Lemma 5.2 implies $A \in \mathbf{C}$.

We have shown that to answer the question whether an AD-class in $\mathbf{A}$ is hereditary it suffices to study the corresponding AD-class in $\mathbf{A} \cap \mathbf{T o p}_{0}$. Since for a 
bireflective subcategory $\mathbf{A} \neq$ Top we have $I_{2} \notin \mathbf{A} \cap \mathbf{T o p}_{0}$, this is precisely the situation examined in the preceding parts of this paper.

Lemma 5.5. If $f: X \rightarrow Y$ is a surjective initial map, $b \in Y$ and $f^{-1}(b)=\{a\}$, then $X_{a} \in \mathrm{CH}\left(Y_{b}\right)$.

Proof. Let $g: Y_{b} \rightarrow X_{a}$ be any map such that $f(g(x))=x$ for any $x \in X_{a}$ (in particular, $g(b)=a)$. We first show that $g$ is continuous.

If $a \in U$ and $U$ is open in $X_{a}$, then there exists an open set $U^{\prime} \subseteq Y$ with $a \in f^{-1}\left(U^{\prime}\right) \subseteq U$. Then $g^{-1}(U) \supseteq g^{-1}\left(f^{-1}\left(U^{\prime}\right)\right)=U^{\prime} \ni b$, hence $g^{-1}(U)$ is open in $Y_{b}$.

We have continuous maps $f, g$ such that $f \circ g=i d_{X_{a}}$. So $f$ is a retraction, thus it is a quotient map and $X_{a} \in \mathrm{CH}\left(Y_{b}\right)$.

Corollary 5.6. Let $X$ be a topological space, $a \in X$ be a point such that $X_{a}$ is $T_{2}$ and $r: X \rightarrow R X_{0}$ be the $T_{0}$-reflection of $X$. Then $X_{a} \in \mathrm{CH}\left(\left(R_{0} X\right)_{b}\right)$, where $b=r(a)$.

Proof. Since $\overline{\{a\}}=\{a\}$, the equivalence class of the point $a$ consists of this single point. Therefore $r^{-1}(b)=\{a\}$ holds for the $\mathbf{T o p}_{0}$-reflection $r$ of $X$. The claim follows now from Lemma 5.5 .

Note that, if $\mathbf{A}$ is none of the categories $\mathbf{T o p}, \mathbf{T o p}_{0}$, then all prime factors belonging to $\mathbf{A}$ are Hausdorff. So we see from the above corollary that, if $\mathbf{B}$ is an $\mathrm{AD}$-class in an epireflective subcategory $\mathbf{A} \neq \mathbf{T o p}, \mathbf{T o p}_{0}$ and $R_{0} \mathbf{B}$ is closed under the formation of prime factors, then $\mathbf{B}$ is closed under the formation of prime factors too.

So our results for arbitrary epireflective subcategories can be subsumed as follows:

Proposition 5.7. Let $\mathbf{A} \neq$ Ind be an epireflective subcategory of Top and $\mathbf{B}$ be an AD-class in $\mathbf{A}$. If $\mathbf{A} \cap \mathbf{T o p}_{0} \subseteq$ Haus or the subcategories $\mathbf{A} \cap \mathbf{T o p}_{0}$ and $\mathbf{B} \cap \mathbf{T o p}_{0}$ fulfill the assumptions of Theorem 3.10 or those of Corollary [6.8, then $\mathbf{B}$ is hereditary if and only if it is closed under the formation of prime factors which belong to $\mathbf{A}$.

In particular we get that $\mathbf{B}$ is closed under the formation of prime factors which are Hausdorff.

\section{AD-Classes and HAD-Classes Containing a PRIme SPACE}

We have shown in Corollary 3.11 that if an HAD-class contains a prime space then it is closed under the formation of prime factors. In connection with this result it seems useful to give some conditions on an HAD-class $\mathbf{B}$ which imply that B contains at least one prime space.

Unfortunately we were able neither to find a counterexample to the claim that every HAD-class (in an epireflective subcategory A of Top with $I_{2} \notin \mathbf{A}$ ) contains a prime space nor to prove this in general.

We have already shown that if an HAD-class $\mathbf{B}$ contains a Hausdorff non-discrete space then it contains a prime space (Proposition 3.13). In this section we provide further sufficient conditions. The main results we obtain are the following: If $\mathbf{B}$ contains a space which is not locally connected, then it contains a prime space (Corollary 6.8). The same holds for non-discrete totally disconnected spaces. 
We also show that an AD-class contains a prime space if and only if it contains the space $C(\alpha)$ for some regular cardinal $\alpha$. At the end of this section we provide some consequences of our results for the lattices of all coreflective subcategories of Top and of some epireflective subcategories of Top.

\subsection{AD-classes containing $C(\alpha)$.}

Definition 6.1. For any infinite cardinal $\alpha$ we denote by $C(\alpha)$ the space on the set $\alpha \cup\{\alpha\}$ such that each $\beta \in \alpha$ is isolated and the sets $B_{\beta}=\{\xi \in \alpha \cup\{\alpha\} ; \xi \geq \beta\}$ for $\beta<\alpha$ form a local base at $\alpha$.

The most important case is the case when $\alpha$ is regular, since for any $\alpha \in \mathrm{Cn}$ there exists a regular cardinal $\beta$ with $\mathrm{CH}(C(\alpha))=\mathrm{CH}(C(\beta)$ ). (Namely, $\beta$ is the cofinality of $\alpha$.)

If $\alpha$ is regular we have a simpler description of the topology of $C(\alpha)$ : A subset $V$ of $C(\alpha)$ is open if either $\alpha \notin V$ or $\operatorname{card}(C(\alpha) \backslash V)<\alpha$. Note that this implies that every injective map $f: C(\alpha) \rightarrow C(\alpha)$ such that $f(\alpha)=\alpha$ is continuous.

In this part we show that an AD-class contains a prime space if and only if it contains some space $C(\alpha)$. We first state two lemmas needed in the proof.

Lemma 6.2. Let $\alpha$ be an infinite regular cardinal. If $Y \prec C(\alpha)$ is a prime space (with the accumulation point $\alpha$ ), then $C(\alpha) \in \mathrm{CH}(Y)$.

Proof. Let $f_{i}, i \in I$, be the family of all injective mappings $f_{i}: \alpha \cup\{\alpha\} \rightarrow \alpha \cup\{\alpha\}$ such that $f_{i}(\alpha)=\alpha$. Let us denote by $X$ the topological space on $\alpha \cup\{\alpha\}$ with the final topology with respect to the family $f_{i}: Y \rightarrow X$. We claim that $X=C(\alpha)$. One of the maps $f_{i}$ is the identity, hence $Y \prec X$ and $\alpha$ is non-isolated in $X$.

Since $Y \prec C(\alpha)$ and all $f_{i}$ 's considered as maps from $C(\alpha)$ to $C(\alpha)$ are continuous, we get $X \prec C(\alpha)$.

To verify that $C(\alpha) \prec X$ we show that any set which is not closed in $C(\alpha)$ is not closed in $X$.

From $X \prec C(\alpha)$ follows that $X$ is a prime space, therefore it suffices to compare the sets not containing its accumulation point $\alpha$. So let $V$ be a subset of $\alpha$ with cardinality $\alpha$ and $\alpha \notin V$. Then there exists an $i \in I$ such that $f_{i}$ maps bijectively the set $\alpha$ to $V$. Since the subset $\alpha$ is not closed in $Y$ (the point $\alpha$ is not isolated), we get that $V$ is not closed in $X$.

Lemma 6.3. Let $\alpha$ be any infinite cardinal. If $Y \prec C(\alpha)$ is a prime space (with the accumulation point $\alpha$ ), then there exists a regular cardinal $\beta$ with $C(\beta) \in \mathrm{CH}(Y)$.

Proof. Let $\beta$ be the cofinality of $\alpha$. There exists a quotient map $q: C(\alpha) \rightarrow C(\beta)$ which maps only the point $\alpha$ to $\beta$. Let $Y^{\prime}$ be the quotient of $Y$ with respect to the same map $q$. Then $Y^{\prime}$ is a prime space, since $q^{-1}(\beta)=\{\alpha\}$ and $\alpha$ is not isolated in $Y$. Moreover, $Y^{\prime} \prec C(\beta)$ and $\beta$ is a regular cardinal, thus $C(\beta) \in \mathrm{CH}\left(Y^{\prime}\right) \subseteq \mathrm{CH}(Y)$ by Lemma 6.2

Proposition 6.4. If an AD-class $\mathbf{B}$ in an epireflective subcategory $\mathbf{A} \neq \mathbf{I n d}$ contains a prime $T_{2}$-space then it contains $C(\alpha)$ for some regular cardinal number $\alpha$.

Proof. Let $P$ be a prime space with the accumulation point $a$. Denote by $\alpha$ the smallest cardinality of a non-closed subset of $P \backslash\{a\}$. Let $C$ be some such subset. 
If $V$ is any subset of $C$ with cardinality smaller than $\alpha$ then it is closed (since $\alpha$ was chosen as the smallest cardinality of a non-closed set). Therefore $C \cup\{a\}$ is a prime subspace of $P$ and it is finer than $C(\alpha)$. (In the case that $\alpha$ is regular it is even homeomorphic, but in either case complements of all basic neighborhoods $B_{\beta}$ of $\alpha$ are closed.)

The claim follows now from Lemma 6.3.

Since every prime $T_{2}$-space is zero-dimensional, Proposition 6.4 could be also deduced from Proposition 6.11. But the proof presented here is more straightforward.

6.2. How to obtain a prime space. We now turn our attention to some conditions which are sufficient to enforce that an HAD-class contains a prime space.

Let us denote by Con the class of all connected spaces. By [9, Satz 21.2.6] its coreflective hull $\mathrm{CH}(\mathbf{C o n})$ consists precisely of sums of connected spaces. An equivalent characterization is that $X \in \mathrm{CH}(\mathbf{C o n})$ if and only if each point of $X$ has an open connected neighborhood.

Proposition 6.5. If $X$ is not a sum of connected spaces then there exists a quotient map $f: X \rightarrow P$, where $P$ is a prime $T_{2}$-space and $P \prec C(\alpha)$.

Proof. Since $X$ does not belong to $\mathrm{CH}($ Con $)$, there exists $a \in X$ such that no open neighborhood of $a$ is connected. This means that for any open neighborhood $U$ of $a$ there exist disjoint open proper subsets $V, W$ of $U$ such that $V \cup W=U$. By transfinite induction we construct a decreasing family $U_{\alpha}$ of open neighborhoods of $a$. We put $U_{0}=X$. For any $\beta$ the neighborhood $U_{\beta}$ can be divided into two disjoint open non-empty sets. Denote by $U_{\beta+1}$ that one which contains $a$. Now suppose that $\beta$ is a limit ordinal and $U_{\gamma}$ is already defined for each $\gamma<\beta$. We put $U_{\beta}:=\bigcap_{\gamma<\beta} U_{\gamma}$ if this set is open. If not, we stop the process and put $\alpha:=\beta$. (We must stop at some ordinal $\beta$, otherwise there would be a proper class of open sets in $X$.)

Thus we get a system $\left(U_{\beta}\right)_{\beta<\alpha}$ of open neighborhoods of $a$ with the following properties: $U_{\beta} \varsubsetneqq U_{\gamma}$ whenever $\beta>\gamma$. For any limit ordinal $\beta<\alpha$ the equality $U_{\beta}=\bigcap_{\gamma<\beta} U_{\gamma}$ holds. The set $U_{\beta} \backslash U_{\beta+1}$ is open for any $\beta<\alpha$, but $\bigcap_{\beta<\alpha} U_{\beta}$ is not open.

Now we define $f: X \rightarrow \alpha \cup\{\alpha\}$ by

$$
f(x)=\sup \left\{\beta \in \alpha: x \in U_{\beta}\right\} .
$$

Recall (Definition 6.1) that a neighborhood base for $C(\alpha)$ at $\alpha$ consists of the sets $B_{\beta}=\{\xi \in \alpha \cup\{\alpha\} ; \xi \geq \beta\}$ for $\beta<\alpha$. We have $f^{-1}\left(B_{\beta}\right)=U_{\beta}, f^{-1}(\beta)=U_{\beta} \backslash U_{\beta+1}$ for any $\beta<\alpha$ and $f^{-1}(\alpha)=\bigcap_{\beta<\alpha} U_{\beta}$. Thus the quotient space w.r.t. the map $f$ is finer than $C(\alpha)$ and the point $\alpha$ is non-isolated in it. Hence it is a prime $T_{2}$-space.

Propositions 6.5 and 3.13 imply that, if there exist an epireflective subcategory $\mathbf{A}$ of Top, $\mathbf{A} \neq \mathbf{I n d}$, and an HAD-class $\mathbf{B}$ in $\mathbf{A}$ not containing a prime space, then $\mathbf{B} \subseteq \mathrm{CH}(\mathbf{C o n})$ and $\mathbf{B}$ contains no non-discrete $T_{2}$-space.

A topological space $X$ is totally disconnected if all components of $X$ are singletons ([6, Notes after section 6.2], [25, Definition 29.1]). The class of totally disconnected spaces forms a quotient-reflective subcategory TD of Top. If a totally disconnected space $X$ is a sum of connected spaces, then $X$ is clearly discrete. 
Corollary 6.6. If $X$ is non-discrete and totally disconnected then there exists a quotient map from $X$ to a prime $T_{2}$-space.

All zero-dimensional spaces $T_{0}$-spaces are totally-disconnected, thus the above corollary applies to the class $\mathbf{Z D}_{\mathbf{0}}$ as well. We will see in Proposition 6.11 that in the case of zero-dimensional spaces this result can be slightly improved, which leads to the description of atoms above Disc in the lattice of coreflective subcategories of the category $\mathbf{Z D}_{\mathbf{0}}$.

We say that a space $X$ is locally connected if for any open neighborhood $U$ of $x$ there is an open neighborhood $V \subseteq U$ of $x$, which is connected (see [6, Problem 6.3.3] or [25], Definition 27.7]). The class of locally connected spaces is a coreflective subcategory of Top.

Lemma 6.7. Let $X$ be a topological space. If $X$ is not locally connected then there exists an open subspace $V$ of $X$ such that $V$ is not a sum of connected spaces.

Proof. If $X$ is not locally connected then there exist a point $x$ and an open neighborhood $V$ of $x$ such that no open neighborhood $U$ of $x$ with $U \subseteq V$ is connected. So $x$ has no open connected neighborhood in the subspace $V$ and $V \notin \mathrm{CH}(\mathbf{C o n})$.

Corollary 6.8. Let $\mathbf{A}$ be an epireflective subcategory of Top with $I_{2} \notin \mathbf{A}$. If $\mathbf{B}$ is an HAD-class in $\mathbf{A}$ and $\mathbf{B}$ contains at least one space which is not locally-connected, then $\mathbf{B}$ is closed under prime factors.

6.3. Lattices of coreflective subcategories. The rest of this section is devoted to showing some new facts concerning the (large) lattice of all coreflective subcategories of Top and of $\mathbf{Z D}$, which follow from the results above or can be shown using similar methods.

Definition 6.9. Let $\alpha$ be a regular cardinal. Then $B(\alpha)$ is the topological space on the set $\alpha \cup\{\alpha\}$ whose open sets are precisely the sets $B_{\beta}=\{\xi \in \alpha \cup\{\alpha\} ; \xi \geq \beta\}$ for $\beta<\alpha$. We will denote the coreflective hull of $B(\alpha)$ in Top by $\mathbf{B}_{\alpha}$.

Many interesting facts about the lattice of all coreflective subcategories of Top can be found in [9, §22] and [10. It is shown that the atoms of this lattice above FG are precisely the subcategories $\mathbf{B}_{\alpha}$. It is also shown that $\mathbf{B}_{\alpha} \subseteq \mathrm{CH}(C(\alpha))$ and $\mathrm{CH}(C(\alpha)) \cap \mathrm{CH}(C(\beta))=\mathbf{F G}$ for any regular cardinals $\alpha \neq \beta$.

Next we show that the minimal elements of the lattice of all coreflective subcategories of Top such that $\mathbf{C} \nsubseteq \mathrm{CH}(\mathbf{C o n})$ are precisely the subcategories $\mathrm{CH}(C(\alpha))$. (Note that the spaces $B(\alpha)$ are connected whereas $C(\alpha) \notin \mathrm{CH}(\mathbf{C o n})$.)

Proposition 6.10. If $\mathbf{C}$ is a subcategory of Top with $\mathbf{C} \nsubseteq \mathrm{CH}(\mathbf{C o n})$, then there exists a regular cardinal $\alpha$ such that $\mathrm{CH}(C(\alpha)) \subseteq \mathbf{C}$.

Proof. If we have $X \in \mathbf{C}$, where $\mathbf{C}$ is coreflective and $X \notin \mathrm{CH}(\mathbf{C o n})$, then by Proposition 6.5 and Lemma 6.3 we get $C(\alpha) \in \mathbf{C}$ for some cardinal $\alpha$.

The subcategory $\mathbf{B}_{\alpha}=\mathrm{CH}(B(\alpha))$ is the smallest coreflective subcategory of Top such that in each space $X \in \mathbf{B}_{\alpha}$ any intersection of less than $\alpha$ open sets is open, and there exists a space $Y \in \mathbf{B}_{\alpha}$ and a system of $\alpha$ open sets in $Y$ with a non-open intersection.

We show that if we have a zero-dimensional space with similar properties then we can obtain a prime $T_{2}$-space from it. Thus the atoms in the lattice of coreflective subcategories of $\mathbf{Z D}$ above the class $\mathbf{F G} \cap \mathbf{Z D}$ have a similar description. The proof of the following proposition is similar to the proof of [22, Proposition 4.4]. 
Proposition 6.11. Let $X$ be a zero-dimensional space and $\alpha$ be the smallest cardinal number such that there exists a system $U_{\beta}, \beta<\alpha$, of open subsets of $X$ with non-open intersection $\bigcap_{\beta<\alpha} U_{\beta}$, but every intersection of less than $\alpha$ open subsets of $X$ is open. Then there exists a prime space $Y \prec C(\alpha)$ and a quotient map $q: X \rightarrow Y$.

Proof. Denote by $\left\{U_{\beta} ; \beta<\alpha\right\}$ the system of $\alpha$ open sets in $X$ whose intersection is not open. We can assume w.l.o.g. that this system is strictly decreasing and all sets $U_{\beta}$ are clopen. (From an arbitrary decreasing system of open sets we obtain a system of clopen sets by choosing any point $a \in \bigcap_{\gamma<\alpha} U_{\gamma} \backslash \operatorname{Int}\left(\bigcap_{\gamma<\alpha} U_{\gamma}\right)$ and choosing a basic neighborhood $U_{\beta}^{\prime}$ with $a \in U_{\beta}^{\prime} \subseteq U_{\beta}$ for each $\beta<\alpha$.) If necessary, we can modify this system in such a way that $U_{0}=X$ and $U_{\beta}=\bigcap_{\gamma<\beta} U_{\gamma}$ for any limit ordinal $\beta<\alpha$.

Define $f: X \rightarrow \alpha \cup\{\alpha\}$ by

$$
f(x)=\sup \left\{\beta \in \alpha: x \in U_{\beta}\right\} .
$$

Let $Y$ be the quotient space with respect to $f$.

The equality $f^{-1}\left(B_{\beta}\right)=U_{\beta}$ holds for any $\beta<\alpha$. Since each $U_{\beta}$ is clopen, we see that $B_{\beta}$ and its complement are open in the quotient topology.

The set $f^{-1}(\alpha)=\bigcap_{\beta<\alpha} U_{\beta}$ is not open, therefore $\{\alpha\}$ is not open. Since the sets $U_{\beta}$ are clopen, all sets $\{\beta\}=f^{-1}\left(U_{\beta} \backslash U_{\beta+1}\right)$ are open in $Y$. Thus $Y$ is indeed a prime space and $Y \prec C(\alpha)$.

Theorem 6.12. Let $\mathbf{A}=\mathbf{Z D}$ or $\mathbf{A}=\mathbf{Z D}_{\mathbf{0}}$. Let $\mathbf{C}$ be a coreflective subcategory (an AD-class) in $\mathbf{A}$ such that $\mathbf{C} \nsubseteq \mathbf{F G} \cap \mathbf{A}$ and $\alpha$ be the smallest cardinal such that there exists a space $X \in \mathbf{C}$ and a system $U_{\beta}, \beta<\alpha$, of open sets in $X$ whose intersection $\bigcap_{\beta<\alpha} U_{\beta}$ is not open. Then there exists a regular cardinal $\alpha$ such that $\mathrm{CH}_{\mathbf{A}}(C(\alpha)) \subseteq \mathbf{C}\left(\right.$ resp. $\left.\mathrm{AD}_{\mathbf{A}}(C(\alpha)) \subseteq \mathbf{C}\right)$.

\section{FURTHER APPLICATIONS}

In this section we study some other questions which are related to HAD-classes.

7.1. HAD-hulls and hereditary coreflective hulls. The aim of this part is to show that if the coreflective hull of $\mathbf{D}$ in $\mathbf{A}$ is hereditary, it is at the same time the HAD-hull of $\mathbf{D}$ in $\mathbf{A}$.

Recall that the coreflective hull $\mathrm{CH}_{\mathbf{A}}(\mathbf{D})$ of $\mathbf{D}$ in $\mathbf{A}$ can be formed by taking all A-extremal quotients of topological sums of spaces from $\mathbf{D}$.

Lemma 7.1. Let $\mathbf{A}$ be an epireflective subcategory of Top with $I_{2} \notin \mathbf{A}$ and $\mathbf{D} \subseteq \mathbf{A}$. Then the prime $T_{2}$-spaces contained in $\mathrm{CH}_{\mathbf{A}}(\mathbf{D})$ and the prime $T_{2}$-spaces contained in $\mathrm{CH}(\mathbf{D})$ are the same. I.e., $\left\{P \in \mathrm{CH}_{\mathbf{A}}(\mathbf{D}), P\right.$ is a prime $T_{2}$-space $\}=\{P \in$ $\mathrm{CH}(\mathbf{D}), P$ is a prime $T_{2}$-space $\}$.

In the case $\mathbf{A}=\mathbf{T o p}_{0}$ we moreover get $\left\{P \in \mathbf{C H}_{\mathbf{A}}(\mathbf{D}), P\right.$ is prime $\}=\{P \in$ $\mathrm{CH}(\mathbf{D}), P$ is prime $\}$.

Proof. Let $P$ be a prime $T_{2}$-space belonging to $\mathrm{CH}_{\mathbf{A}}(\mathbf{D})$. There is an A-extremal epimorphism $e: A \rightarrow P$, where $A$ is a sum of spaces from $\mathbf{D}$, which can be factorized 
as $m \circ q$ with $q$ a quotient map and $m$ an injective continuous map.

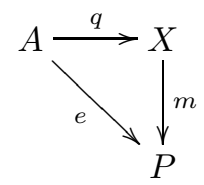

Since $m: X \rightarrow P$ is an injective map, $X$ is either discrete or a prime $T_{2}$-space. Thus $X \in \mathbf{Z D}_{\mathbf{0}} \subseteq \mathbf{A}$, and $m$ is an $\mathbf{A}$-monomorphism. Since $e$ is A-extremal epimorphism, we obtain that $m$ is an isomorphism and $P \in \mathrm{CH}(\mathbf{D})$.

Any prime space is $T_{0}$. Therefore the second part is clear from the equality $\mathrm{CH}_{\mathbf{A}}(\mathbf{D})=\mathrm{CH}(\mathbf{D}) \cap \mathbf{A}$, which holds for $\mathbf{A}=\mathbf{T o p}_{0}$.

Theorem 7.2. Let $\mathbf{A}$ be an epireflective subcategory of Top with $I_{2} \notin \mathbf{A}$ and $\mathbf{D} \subseteq \mathbf{A}$. If $\mathrm{CH}_{\mathbf{A}}(\mathbf{D})$ is hereditary then $\mathrm{CH}_{\mathbf{A}}(\mathbf{D})=\mathrm{CH}(\mathbf{D}) \cap \mathbf{A}$.

Proof. The inclusion $\mathrm{CH}(\mathbf{D}) \cap \mathbf{A} \subseteq \mathrm{CH}_{\mathbf{A}}(\mathbf{D})$ holds for any $\mathbf{D} \subseteq \mathbf{A}$. We show the opposite inclusion.

If $\mathrm{CH}_{\mathbf{A}}(\mathbf{D})$ is a hereditary coreflective subcategory of $\mathbf{A}$, then it is closed under the formation of prime factors (see [4, Theorem 1]). Let $Y \in \mathrm{CH}_{\mathbf{A}}(\mathbf{D})$. If $\mathbf{A} \subseteq \mathbf{T o p}_{1}$ then any prime factor $Y_{a}$ of $Y$ is $T_{2}$. All of them belong to $\mathrm{CH}_{\mathbf{A}}(\mathbf{D})$. According to Lemma 7.1 prime $T_{2}$-spaces in $\mathrm{CH}_{\mathbf{A}}(\mathbf{D})$ and $\mathrm{CH}(\mathbf{D})$ are the same. As $Y$ is a quotient of spaces $Y_{a}$ belonging to $\mathrm{CH}(\mathbf{D})$, we get $Y \in \mathrm{CH}(\mathbf{D})$. A.

In the case $\mathbf{A}=\mathbf{T o p}_{0}$ the equality $\mathrm{CH}_{\mathbf{A}}(\mathbf{D})=\mathrm{CH}(\mathbf{D}) \cap \mathbf{A}$ holds for any $\mathbf{D} \subseteq$

Corollary 7.3. Let $\mathbf{A}$ be an epireflective subcategory of Top with $I_{2} \notin \mathbf{A}$ and $\mathbf{D} \subseteq \mathbf{A}$. If $\mathrm{CH}_{\mathbf{A}}(\mathbf{D})$ is hereditary then $\mathrm{CH}_{\mathbf{A}}(\mathbf{D})=\operatorname{AD}_{\mathbf{A}}(\mathbf{D})=\operatorname{HAD}_{\mathbf{A}}(\mathbf{D})$.

This corollary implies that the results we proved about HAD-hulls in A can be applied in the case of hereditary coreflective hulls in $\mathbf{A}$ as well.

E.g., if $\mathbf{D} \subseteq \mathbf{A}$ is a set of spaces and $\mathbf{B}=\mathrm{CH}_{\mathbf{A}}(\mathbf{D})$ is hereditary, then by Corollary 7.3 it fulfills the assumptions of Lemma 4.10 and we get existence of a space $B$ with $\mathbf{B}=\mathrm{CH}_{\mathbf{A}}(B)$ in this case.

7.2. Coreflective hull of a map invariant hereditary class need not be hereditary. Finally, we turn our attention to another question. Relatively little is known about conditions on a class of spaces which ensure the heredity of the coreflective hull (AD-hull) of this class. V. Kannan has a result saying that if $\mathbf{B}$ is a hereditary family closed under the formation of spaces with finer topologies then the coreflective hull $\mathrm{CH}(\mathbf{B})$ of $\mathbf{B}$ in Top is hereditary as well ([16, Remark 2.4.4(6)]). Our Theorem 4.11 yields a kind of such condition, too. We next present a well-known example of classes $\mathbf{B}$ such that $\mathrm{CH}(\mathbf{B})$ is hereditary.

Example 7.4. Let $\alpha$ be an infinite cardinal and $\mathbf{G}_{\alpha}$ be the class of all spaces with cardinality at most $\alpha$. These classes are hereditary, map invariant (i.e., closed under continuous images) and closed under the formation of prime factors. The coreflective hull of $\mathbf{G}_{\alpha}$ is hereditary for each $\alpha$. Spaces from the coreflective hull of $\mathbf{G}_{\alpha}$ are called $\alpha$-generated and the subcategory of all $\alpha$-generated spaces is denoted $\operatorname{Gen}(\alpha)$.

On the other hand, let $\mathbf{B}$ be a class of topological spaces which is map invariant and closed under prime factors. It is easy to show that if $\mathbf{B}$ contains an infinite 
space, then either $\mathbf{B}=$ Top or $\mathbf{B}=\mathbf{G}_{\alpha}$ for some cardinal $\alpha$. If $\mathbf{B}$ consists of finite spaces only, then $\mathrm{CH}(\mathbf{B})$ is either FG or Disc.

It is natural to ask whether we can somehow weaken the above mentioned properties of the classes $\mathbf{G}_{\alpha}$ in such a way, that for every class $\mathbf{B}$ with these properties the coreflective hull $\mathbf{C H}(\mathbf{B})$ of $\mathbf{B}$ in Top is hereditary.

One possible weakening is replacing the condition that $\mathbf{B}$ is map invariant by divisibility. We can construct easily an example showing that for such a class $\mathrm{CH}(\mathbf{B})$ need not be hereditary in general.

Example 7.5. Let $\mathbf{B}$ consist of all quotients of the space $C(\omega)$ and of all discrete (at most) countable spaces. This class is clearly divisible. Every space in $\mathbf{B}$ is prime or discrete, hence $\mathbf{B}$ is closed under the formation of prime factors. A subspace of a prime space $P$ is either a discrete space or a quotient of $P$, thus $\mathbf{B}$ is hereditary.

The coreflective hull $\mathrm{CH}(\mathbf{B})=\mathrm{CH}(C(\omega))=$ Seq is not hereditary.

Another possible weakening is omitting the closedness under prime factors. We show in the rest of this section that there exists a class $\mathbf{B}$ which is hereditary and map invariant but $\mathrm{CH}(\mathbf{B})$ is not hereditary.

We start with two easy examples.

Example 7.6. Let $\mathbf{B}$ be the class of all continuous images of the space $B(\omega)$. The class $\mathbf{B}$ is hereditary and map invariant, but $\mathrm{CH}(\mathbf{B})=\mathrm{CH}(B(\omega))$ is not hereditary. (This follows from the fact that the prime factor $(B(\omega))_{\omega}$ is $C(\omega)$ and $C(\omega) \notin$ $\mathrm{CH}(B(\omega))$.)

It is known, that if $\mathbf{A}$ is a map invariant class of topological spaces then $\mathrm{CH}(\mathbf{A})$ coincides with the class $\mathbf{A}_{\text {gen }}$ of $\mathbf{A}$-generated spaces (see [9, §21] or [10]). A topological space $X$ is said to be A-generated if $U \subseteq X$ is closed whenever $U \cap V$ is closed in $V$ for every subspace $V$ of $X$ which belongs to A. The subcategory $\operatorname{Gen}(\alpha)$ and the class of $k$-spaces used in Example 3.18 are examples of such categories.

We denote the cardinality of the topology of a space $X$ by $o(X)$ (in accordance with [14). For any cardinal $\alpha$ let us denote by $\mathbf{A}_{\alpha}$ the class of all topological spaces such that $o(X)<2^{\alpha}$. This class is hereditary and map invariant. Its coreflective hull (i.e., the class of all $\mathbf{A}_{\alpha}$-generated spaces) will be denoted by $\mathbf{C}_{\alpha}$.

Note that $o(B(\omega))=\omega$, thus $\mathrm{CH}(\mathbf{B}) \subseteq \mathbf{C}_{\omega}$ holds for the category $\mathbf{B}$ from Example 7.6

Example 7.7. We show that $\mathbf{A}_{\omega_{0}}$ is not closed under the formation of prime factors and consequently it is not hereditary.

Let $X$ be a countable topological space with the cofinite topology. Clearly, $o(X)=\omega_{0}$, thus $X \in \mathbf{A}_{\omega_{0}}$. But the prime factor $X_{a}$ of $X$ is homeomorphic to $C(\omega)$. Only the finite subspaces of $C(\omega)$ belong to $\mathbf{A}_{\omega_{0}}$. Thus the point $\omega$ is isolated in each subspace belonging to $\mathbf{A}_{\omega_{0}}$ and $C(\omega) \notin \mathbf{C}_{\omega_{0}}$.

Note that by Proposition 3.13 in every non-discrete Hausdorff space $X$ we have infinitely many disjoint open subsets in the subspace $Y$ constructed in the proof of this proposition. Therefore $o(X) \geq \mathfrak{c}$. This implies $\mathbf{A}_{\omega_{0}} \cap$ Haus $\subseteq$ Disc and, consequently, $\mathbf{C}_{\omega_{0}} \cap$ Haus $=$ Disc.

Note that, since the space constructed in the above example is $T_{1}$, we also obtain that $\mathrm{CH}_{\mathbf{A}}\left(\mathbf{A}_{\alpha} \cap \mathbf{A}\right)$ is not hereditary for $\mathbf{A}=\mathbf{T o p}_{0,1}$.

It is quite natural to look for a Hausdorff example after we have constructed a $T_{1}$-space with the required properties. We have already seen that such an example 
cannot be found in the subcategory $\mathbf{C}_{\omega_{0}}$. We were able to construct a Hausdorff example only under the assumption $2^{\omega_{1}}=2^{\mathfrak{c}}$ (which is valid under $\mathrm{CH}$ ).

Example 7.8 $\left(2^{\omega_{1}}=2^{\mathfrak{c}}\right)$. Suppose $2^{\omega_{1}}=2^{\mathfrak{c}}$. Let $X$ be the topological space on the set $\mathbb{R}$ with the topology $\mathcal{T}=\left\{U \backslash A ; U\right.$ is open in $\mathbb{R}$ and card $\left.A \leq \omega_{0}\right\}$. Clearly, $o(X)=o(\mathbb{R}) \cdot \operatorname{card}\{A \subseteq \mathbb{R} ; A$ is countable $\}=\mathfrak{c} \cdot \mathfrak{c}^{\omega_{0}}=\mathfrak{c}$. Thus $X \in \mathbf{A}_{\mathfrak{c}}$.

We claim that, for any $a \in X$, the prime factor $X_{a}$ does not belong to $\mathbf{C}_{\mathfrak{c}}$. Indeed, if $a \in V$ and $V$ is a subspace of $X_{a}$ such that $V \in \mathbf{A}_{\mathfrak{c}}$, then card $V=\omega_{0}$ (otherwise $V$ contains a discrete subspace $V \backslash\{a\}$ of cardinality $\omega_{1}$ and $\left.o(V)=2^{\omega_{1}}=2^{\mathfrak{c}}\right)$. At the same time $a \notin \overline{V \backslash\{a\}}$ (since $\{a\} \cup(\mathbb{R} \backslash V)$ is a neighborhood of $a$ ). We see that $a$ is isolated in all subspaces of $X_{a}$ belonging to $\mathbf{A}_{\mathfrak{c}}$, but $a$ is not isolated in $X_{a}$, thus $X_{a} \notin \mathbf{C}_{\mathfrak{c}}$.

Example 7.9. After we have shown that $\mathbf{C}_{\alpha}$ is not hereditary for some $\alpha$, we can be interested in finding a concrete example of a space from $\mathbf{C}_{\alpha}$ and its subspace which is not in $\mathbf{C}_{\alpha}$. Such an example can be found with the help of the operation $\triangle$.

Suppose that $X \in \mathbf{C}_{\alpha}$ is such a space that $X_{a} \notin \mathbf{C}_{\alpha}$. Let $Y:=X \triangle_{a} X$. Clearly, $Y \in \mathbf{C}_{\alpha}$. Recall that $X_{(X, a)}^{a}$ is the subspace on the set $\{(a, a)\} \cup(X \backslash\{a\}) \times(X \backslash\{a\})$. Since $X_{a} \notin \mathbf{C}_{\alpha}$ and $X_{a}$ is a quotient of $X_{(X, a)}^{a}$, we get that the subspace $X_{(X, a)}^{a} \notin \mathbf{C}_{\alpha}$ as well.

Note that, since the subcategories Top $_{1}$, Haus are closed under $\triangle$, if we start with the space $X$ from Example 7.7 (or Example 7.8), the resulting space $Y$ will be $T_{1}$ (resp. Hausdorff) as well.

Acknowledgement. I would like to thank H. Herrlich and J. Činčura for their help and lots of useful comments while preparing this paper. Some parts of this paper were prepared during my stay at Universität Bremen. I am grateful to the members of the KatMAT research group for their hospitality and to DAAD for the financial support.

\section{REFERENCES}

[1] Adámek, J., Herrlich, H., Strecker, G.: Abstract and Concrete Categories. Wiley, New York (1990). Available online at http://katmat.math.uni-bremen.de/acc/acc.pdf

[2] Arhangel'skiı̌, A.V., Franklin, S.P.: Ordinal invariants for topological spaces. Michigan Math. J. 15, 313-320 (1968)

[3] Činčura, J.: Heredity and coreflective subcategories of the category of topological spaces. Appl. Categ. Structures 9, 131-138 (2001)

[4] Činčura, J.: Hereditary coreflective subcategories of categories of topological spaces. Appl. Categ. Structures 13, 329-342 (2005)

[5] Cook, H.: Continua which admit only the identity mapping onto non-degenerate subcontinua. Fund. Math. 60, 241-249 (1967)

[6] Engelking, R.: General Topology. PWN, Warsaw (1977)

[7] Giuli, E., Hušek, M.: A counterpart of compactness. Boll. Unione Mat. Ital., VII. Ser., B 11(3), 605-621 (1997)

[8] de Groot, J.: Groups represented by homeomorphism groups. I. Math. Ann. 138, 80-102 (1959)

[9] Herrlich, H.: Topologische Reflexionen und Coreflexionen. Springer, Berlin (1968). Lecture Notes in Math. 78

[10] Herrlich, H.: Limit-operators and topological coreflections. Trans. Amer. Math. Soc. 146, 203-210 (1969)

[11] Herrlich, H., Hušek, M.: Some open categorical problems in Top. Appl. Categ. Structures 1, $1-19(1993)$ 
[12] Herrlich, H., Strecker, G.: Category Theory. Heldermann, Berlin (1979)

[13] Hušek, M.: Products of quotients and of $k^{\prime}$-spaces. Comment. Math. Univ. Carolinae 12, 61-68 (1971)

[14] Juhász, I.: Cardinal functions in topology - ten years later. Math. Centre Tracts, Amsterdam (1980)

[15] Kannan, V.: Pairs of topologies with same family of continuous self-maps. In: H. Herrlich, G. Preuss (eds.) Categorical topology, Proceedings of the International Conference, pp. 176184. Springer, Berlin (1979)

[16] Kannan, V.: Ordinal invariants in topology. Mem. Amer. Math. Soc. 245 (1981)

[17] Kannan, V., Rajagopalan, M.: Constructions and applications of rigid spaces. I. Adv. Math. 29, 89-130 (1978)

[18] Lukács, G.: A convenient subcategory of Tych. Appl. Categ. Structures 12, 263-267 (2004)

[19] Marny, T.: On epireflective subcategories of topological categories. General Topology Appl. 10, 175-181 (1979)

[20] Michael, E.: On $k$-spaces and $k_{R}$-spaces and $k(X)$. Pacif. J. Math. 47(2), 487-498 (1973)

[21] Nakagawa, R.: Categorial topology. In: K. Morita, J.I. Nagata (eds.) Topics in general topology, pp. 563-623. North-Holland (1989)

[22] Sleziak, M.: Subspaces of pseudoradial spaces. Math. Slov. 53, 505-514 (2003)

[23] Sleziak, M.: On hereditary coreflective subcategories of Top. Appl. Categ. Structures 12, 301-317 (2004)

[24] Todorčević, S., Uzcátegui, C.: Analytic k-spaces. Topology and its Applications 146-147, $511-526(2005)$

[25] Willard, S.: General topology. Addison-Wesley, Massachussets (1970)

KAGDM FMFi UK, Mlynská dolina, 84248 Bratislava, Slovakia

E-mail address: sleziak@mph.uniba.sk 